\title{
Full-discrete adaptive FEM for quasi-parabolic integro-differential PDE-constrained optimal control problem
}

\section{Wanfang Shen}

\section{"Correspondence:}

wfsrose@163.com

School of Mathematic and

Quantitative Economics, Shandong

University of Finance and

Economics, Shungeng Road, Jinan, 250014, China

\section{Springer}

\begin{abstract}
In this paper, we develop a full-discrete adaptive FEM for a quasi-parabolic integro-differential PDE-constrained optimal control problem. Firstly, we present weak formulations and optimal conditions and, by using the backward Euler scheme, we deduce equivalent a posteriori error estimators. Then we derive lower and upper bounds for the error estimates by bubble function techniques and use these indicators in adaptive finite element schemes. Furthermore, we carry out some numerical experiments to test our theoretical results.
\end{abstract}

Keywords: full-discrete adaptive FEM; equivalent a posteriori error estimates; linear quasi-parabolic integro-differential PDE; optimal control problem; optimality conditions; constrained control

\section{Introduction}

In many applications such as mechanics, heat conduction in materials with memory, and so on, models are often described by quasi-parabolic integro-differential PDEs (IDPDEs). In any subdomain, these models maintain conservation of energy at any moment. In [1], the authors presented the existence and uniqueness of the solution to a quasi-parabolic IDPDE. In [2], the authors studied the finite element methods (FEMs) for quasi-parabolic IDPDE problems.

For classic linear PDE-constrained optimal control problems (OCPs), such as elliptic, parabolic, and hyperbolic equation constrained OCPs. there has been much work on the existence and optimality conditions (see [3]). In [4-11], the authors have already carefully studied the FEM approximation and obtained a priori error estimates. Recently, in [12, 13], the authors concentrated themselves on OCPs governed by IDPEDs such as elliptic, parabolic, and quasi-parabolic IDPDEs. However, although we often encounter those control problems in many practical applications, as far as we know, there has been little study on full-discrete adaptive finite element methods (AFEMs) for quasi-parabolic IDPDE constrained OCP.

As is well known, AFEM can efficiently decrease the computational work among different FEMs in the literature, and it has become one of the main methods in developing FEMs in real scientific and engineering applications. The principle of this method is to use the a posteriori error estimate indicator to guide local mesh refinement in computation;

(c) 2016 Shen. This article is distributed under the terms of the Creative Commons Attribution 4.0 International License (http://creativecommons.org/licenses/by/4.0/), which permits unrestricted use, distribution, and reproduction in any medium, provided you give appropriate credit to the original author(s) and the source, provide a link to the Creative Commons license, and indicate if changes were made. 
in such a way denser meshes are only used where the value of the indicator is larger and the solution is more singular, and consequently the degree of freedoms may be greatly reduced on those only locally refined meshes. One of the keys to make AFEM work is that the value of the error estimate indicator has to be highly positively correlated to the singularity of the solutions to be approximated. So the quality of the a posteriori error indicators is vital for effectiveness of AFEM [14]. In [15-18], the authors present the effectiveness of AFEM and carry out tests to illustrate that AFEM can indeed reduce computational work for PDE-constrained OCP.

In the following, we investigate the following quasi-parabolic IDPDE-constrained OCP:

$$
\min _{u \in X, u(t) \in K} J(u, y(u))=\left\{\frac{1}{2} \int_{0}^{T}\left\|y-z_{d}\right\|_{0, \Omega}^{2} d t+\frac{\alpha}{2} \int_{0}^{T}\|u\|_{0, \Omega}^{2} d t\right\}
$$

subject to

$$
\begin{cases}y_{t}-\operatorname{div}\left(A \nabla y_{t}+D \nabla y+\int_{0}^{t} C(t, \tau) \nabla y(\tau) d \tau\right)=f+u, & (x, t) \in \Omega \times(0, T], \\ y=0, & (x, t) \in \partial \Omega \times[0, T], \\ \left.y\right|_{t=0}=y_{0}, & x \in \Omega,\end{cases}
$$

where $\alpha$ is a positive constant, $u=u(x, t)$ is the control, $y=y(x, t)$ is the state, $f=f(x, t)$, $z_{d}(x, t)$, and $y_{0}=y_{0}(x)$ are some suitable functions, $A=A(x)=\left(a_{i, j}(\cdot)\right)_{n \times n}, D=D(x)=$ $\left(d_{i, j}(\cdot)\right)_{n \times n} \in\left(C^{\infty}(\bar{\Omega})\right)^{n \times n}$, there exists a constant $c>0$ such that $A, D$ satisfy

$$
X^{t} A X \geq c\|X\|_{R^{n}}^{2}, \quad X^{t} D X \geq c\|X\|_{R^{n}}^{2}, \quad \forall X \in R^{n},
$$

and $C=C(x, t, \tau)=\left(c_{i, j}(x, t, \tau)\right)_{n \times n} \in\left(C^{\infty}\left(0, T ; L^{\infty}(\bar{\Omega})\right)\right)^{n \times n}$. Here $\Omega, X, K$ are the sets defined in Section 2.

In this paper, the a posteriori error indicators are presented for AFEM approximation of (1.1)-(1.2). For this control problem, by carrying out a very detailed analysis, we obtain the a posteriori error estimates for the full-discrete AFEM on multimeshes. Here we also employ and extend the existing techniques in $[12,13,19-21]$ to (1.1)-(1.2).

The outline of the paper is as follows. In Section 2, we present the weak formulation and optimal conditions for (1.1)-(1.2). In Section 3, the semidiscrete and full-discrete FEM approximation forms for (1.1)-(1.2) are established. In Section 4, we derive upper and lower a posteriori error bounds for (1.1)-(1.2). Finally, we carry out some numerical experiments to show the effectiveness of the indicators derived in Section 4.

\section{Model problem and the optimal conditions}

In this paper, $\Omega$ is a bounded open set with Lipschitz boundary $\partial \Omega$, and $\Omega_{U}$ is another bounded open set in $R^{d}$, where $1 \leq d \leq 3$.

In the following, we use the standard notation $W^{m, q}(\Omega)$ for Sobolev spaces on $\Omega$ equipped with norm $\|\cdot\|_{m, q, \Omega}$, and seminorm $|\cdot|_{m, q, \Omega}$. We denote $W_{0}^{m, q}(\Omega)=\{w \in$ $\left.W^{m, q}(\Omega):\left.w\right|_{\partial \Omega}=0\right\}$. For convenience, we use $H^{m}(\Omega)\left(H_{0}^{m}(\Omega)\right)$ to denote $W^{m, 2}(\Omega)$ $\left(W_{0}^{m, 2}(\Omega)\right)$ equipped with norm $\|\cdot\|_{m, \Omega}$ and seminorm $|\cdot|_{m, \Omega}$. The Banach space of all $L^{s}$ integrable functions $\left((0, S) \rightarrow W^{m, q}(\Omega)\right)$ is denoted by $L^{s}\left(0, S ; W^{m, q}(\Omega)\right)$, which is equipped with norm $\|v\|_{L^{s}\left(0, S ; W^{m, q}(\Omega)\right)}=\left(\int_{0}^{S}\|v\|_{W^{m, q}(\Omega)}^{s} d t\right)^{\frac{1}{s}}$ for $s \in[1, \infty)$ and with the standard modification for $s=\infty$. In the same way, we also define the spaces $H^{1}\left(0, S ; W^{m, q}(\Omega)\right)$ and $C^{k}\left(0, S ; W^{m, q}(\Omega)\right)$. In [22], we can find the details. 
Throughout the paper, we denote by $c$ or $C$ general positive constants independent of any unknowns and mesh parameters.

Let $V=H_{0}^{1}(\Omega)$ and $U=L^{2}(\Omega)$; as the state and control spaces, we take $W=L^{2}(0, T ; V)$ and $X=L^{2}(0, T ; U)$.

Also, we let $K=\left\{u(t) \in L^{2}(\Omega): \int_{\Omega} u(t) \geq 0, \forall t \in[0, T]\right\}$ be a closed convex subset of $U$, and as the control set, we take $U_{a d}=\{u \in X, u(t) \in K\}$.

In order to present the weak formulation and regularity of the solution of (1.1)-(1.2), we first introduce the following bilinear forms: $\forall z, w \in V \times V$,

$$
\begin{aligned}
& a(z, w)=(A \nabla z, \nabla w), \quad d(z, w)=(D \nabla z, \nabla w), \\
& c(t, \tau ; z, w)=(C(t, \tau) \nabla z, \nabla w), \quad c_{t}(t, \tau ; z, w)=\left(C_{t}(t, \tau) \nabla z, \nabla w\right),
\end{aligned}
$$

where $C_{t}$ is the partial derivative of $C(x, t, \tau)$.

We suppose that these bilinear forms satisfy the following condition: there exist constants $c>0$ and $C>0$ such that

(a) $\quad a(z, z) \geq c\|z\|_{1, \Omega}^{2}, \quad d(z, z) \geq c\|z\|_{1, \Omega}^{2}, \quad \forall z \in V$,

(b) $\quad|a(z, w)| \leq C\|z\|_{1, \Omega}\|w\|_{1, \Omega}, \quad|d(z, w)| \leq C\|z\|_{1, \Omega}\|w\|_{1, \Omega}, \quad \forall z, w \in V$,

(c) $|c(t, \tau ; z, w)| \leq C\|z\|_{1, \Omega}\|w\|_{1, \Omega}$,

$$
\left|c_{t}(t, \tau ; z, w)\right| \leq C\|z\|_{1, \Omega}\|w\|_{1, \Omega}, \quad \forall z, w \in V, t, \tau \in[0, T] .
$$

Therefore, (1.1)-(1.2) can be restated as the $Q C P$

$$
\min _{u \in X, u(t) \in K} J(u, y(u))
$$

subject to

$$
\left\{\begin{array}{l}
\left(y_{t}, w\right)+a\left(y_{t}, w\right)+d(y, w)+\int_{0}^{t} c(t, \tau ; y(\tau), w) d \tau \\
\quad=(f+u, w), \quad \forall w \in V, t \in(0, T], \\
\left.y\right|_{t=0}=y^{0},
\end{array}\right.
$$

where (2.3) is the weak form of the state equation (1.2). From $[1,2]$ we know that there is at least one solution $y$ of (2.3) that satisfies $y \in N(0, T)=\left\{y: y \in L^{2}\left(0, T ; H_{0}^{1}(\Omega)\right), y_{t} \in\right.$ $\left.L^{2}\left(0, T ; H^{1}(\Omega)\right)\right\}$.

From [13] we know that the OCP (2.2)-(2.3) has a unique solution $(y, u) \in N(0, T) \times X$, and $(y, u)$ is the solution of OCP iff there exists a costate $p \in N(0, T)$ such that the triple $(y, p, u)$ satisfies the following optimal conditions QCP-OPT:

$$
\left\{\begin{array}{l}
\left(y_{t}, w\right)+a\left(y_{t}, w\right)+d(y, w)+\int_{0}^{t} c(t, \tau ; y(\tau), w) d \tau \\
\quad=(f+u, w), \quad \forall w \in V, t \in(0, T], \\
\left.y\right|_{t=0}=y_{0}(x), \\
-\left(q, p_{t}\right)-a\left(q, p_{t}\right)+d(q, p)+\int_{t}^{T} c(\tau, t ; q, p(\tau)) d \tau \\
\quad=\left(y-z_{d}, q\right), \quad \forall q \in V, t \in[0, T), \\
\left.p\right|_{t=T}=0, \\
\int_{0}^{T}(\alpha u+p, v-u) d t \geq 0, \quad \forall v \in X, v(t) \in K .
\end{array}\right.
$$




\section{Full-discrete FEM approximation}

We now consider the full-discrete FEM approximation to (1.1)-(1.2). For simplicity, here we only consider triangular conforming elements and suppose that $\Omega^{h}=\Omega$.

Let $T^{h}$ and $T_{U}^{h}$ be two different polygonal approximations to $\Omega^{h}$. On $T^{h}$, we set the finite element subset $S^{h}$ of $C\left(\bar{\Omega}^{h}\right)$ that satisfies: $\forall \chi \in S^{h}, \forall \tau \in T^{h},\left.\chi\right|_{\tau}$ is an $m$-polynomial, where $m \geq 1$ is the degree of the polynomials. Let $V^{h}=\left\{v_{h} \in S^{h}: v_{h}\left(P_{i}\right)=0, i=1, \ldots, J\right\}$ and $W^{h}=$ $L^{2}\left(0, T ; V^{h}\right)$, where $P_{i}$ is the boundary vertex of the domain $T^{h}(i=1, \ldots, J)$. Obviously, we have $V^{h} \subset V, W^{h} \subset W$. On $T_{U}^{h}$, we set the finite element subset $U^{h}$ in $L^{2}\left(\Omega^{h}\right)$ that satisfies: $\forall \chi \in U^{h}, \forall \tau_{U} \in T_{U}^{h},\left.\chi\right|_{\tau_{U}}$ is an $m$-polynomial $(m \geq 1)$. Let $X^{h}=L^{2}\left(0, T ; U^{h}\right)$. Note that we do not require the continuity for $U^{h}$. Then we obviously have $X^{h} \subset X$.

Let the diameter of the maximal circumcircle of an element $\tau\left(\tau_{U}\right)$ in $T^{h}\left(T_{U}^{h}\right)$ be denoted by $h_{\tau}\left(h_{\tau_{U}}\right)$, and the diameter of the maximal inscribed circle of element $\tau\left(\tau_{U}\right)$ in $T^{h}\left(T_{U}^{h}\right)$ be denoted by $\rho_{\tau}\left(\rho_{\tau_{U}}\right)$. Suppose that there exists a positive constant $R$ such that $1 \leq \max _{\tau \in T^{h}}\left(\frac{h_{\tau}}{\rho_{\tau}}\right) \leq R$ and $1 \leq \max _{\tau_{U} \in T_{U}^{h}}\left(\frac{h_{\tau_{U}}}{\rho_{\tau_{U}}}\right) \leq R$, and let $h=\max _{\tau \in T^{h}} h_{\tau}$ and $h_{U}=$ $\max _{\tau_{U} \in T_{U}^{h}} h_{\tau_{U}}$.

Here piecewise constant FE space is used for the approximation of the control due to the limited regularity of the optimal control (normally, $H^{1}$ ). We will use higher-order FE spaces when approximating the state and costate. We denote all 0 -order polynomials on $\tau_{U}$ by $P_{0}\left(\tau_{U}\right)$ and always set $X^{h}=\left\{u \in X:\left.u(x, t)\right|_{x \in \tau_{U}} \in P_{0}\left(\tau_{U}\right), \forall t \in[0, T]\right\}$ and $K^{h}=\left\{u_{h}(t) \in\right.$ $\left.U^{h}: \int_{\Omega} u_{h}(t) \geq 0\right\}$.

Then we can easily present the semidiscrete FEM approximation $(O C P)^{h}$ for $(O C P)$ as follows:

$$
\min _{u_{h} \in X^{h}, u_{h}(t) \in K^{h}}\left\{\frac{1}{2} \int_{0}^{T}\left\|y_{h}-z_{d}\right\|_{0, \Omega}^{2} d t+\frac{\alpha}{2} \int_{0}^{T}\left\|u_{h}\right\|_{0, \Omega}^{2} d t\right\}
$$

subject to

$$
\left\{\begin{array}{l}
\left(\frac{\partial}{\partial t} y_{h}, w_{h}\right)+a\left(\frac{\partial}{\partial t} y_{h}, w_{h}\right)+d\left(y_{h}, w_{h}\right)+\int_{0}^{t} c\left(t, \tau ; y_{h}(\tau), w_{h}\right) d \tau \\
\quad=\left(f+u_{h}, w_{h}\right), \quad \forall w_{h} \in V^{h}, t \in(0, T], \\
\left.y_{h}\right|_{t=0}=y_{0}^{h},
\end{array}\right.
$$

where $y_{h} \in W^{h}$, and $y_{0}^{h} \in V^{h}$ is an approximation of $y_{0}$.

Following from [3] and (2.4), we have that there exists a unique solution $\left(y_{h}, u_{h}\right) \in W^{h} \times$ $K^{h}$ of $(O C P)^{h}$, and we know that a pair $\left(y_{h}, u_{h}\right)$ is a solution of $(O C P)^{h}$ iff there exists a costate $p_{h} \in W^{h}$ and the triple $\left(y_{h}, p_{h}, u_{h}\right)$ satisfies the optimality conditions $(Q C P-O P T)^{h}$ as follows:

$$
\left\{\begin{array}{l}
\left(\frac{\partial}{\partial t} y_{h}, w_{h}\right)+a\left(\frac{\partial}{\partial t} y_{h}, w_{h}\right)+d\left(y_{h}, w_{h}\right)+\int_{0}^{t} c\left(t, \tau ; y_{h}(\tau), w_{h}\right) d \tau \\
\quad=\left(f+u_{h}, w_{h}\right), \quad \forall w_{h} \in V^{h}, \\
y_{h}(x, 0)=y_{0}^{h}(x), \\
-\left(q_{h}, \frac{\partial}{\partial t} p_{h}\right)-a\left(q_{h}, \frac{\partial}{\partial t} p_{h}\right)+d\left(q_{h}, p_{h}\right)+\int_{t}^{T} c\left(\tau, t ; q_{h}, p_{h}(\tau)\right) d \tau \\
\quad=\left(y_{h}-z_{d}, q_{h}\right), \quad \forall q_{h} \in V^{h}, \\
p_{h}(x, T)=0, \\
\left(\alpha u_{h}+p_{h}, v_{h}-u_{h}\right) \geq 0, \quad \forall v_{h} \in K^{h} .
\end{array}\right.
$$


Now we use the backward Euler scheme in time to construct the full-discrete FEM approximation of (3.3) and obtain its full-discrete form. For convenience, we suppose that $\Omega$ is a polygonal area with boundary $\partial \Omega$.

Let $0=t_{0}<t_{1}<\cdots<t_{N}=T, k_{i}=t_{i}-t_{i-1}, i=1,2, \ldots, N, k=\max _{1 \leq i \leq N}\left\{k_{i}\right\}$. For $i=$ $1,2, \ldots, N$, we similarly to $V^{h}$ construct the FE space $V_{i}^{h} \subset H_{0}^{1}(\Omega)$ on mesh $T_{i}^{h}$. We also construct the FE space $U_{i}^{h} \subset L^{2}(\Omega)$ on mesh $\left(T_{U}^{h}\right)_{i}$. Here we denote the maximal diameter of the element $\tau^{i}\left(\tau_{U}^{i}\right)$ in $T_{i}^{h}\left(\left(T_{U}^{h}\right)_{i}\right)$ by $h_{\tau^{i}}\left(h_{\tau_{U}^{i}}\right)$. Then we define the mesh functions $\tau(\cdot)$ and $\tau_{U}(\cdot)$ by $\left.\tau(t)\right|_{t \in\left(t_{i-1}, t_{i}\right]}=\tau^{i}$ and $\left.\tau_{U}(t)\right|_{t \in\left(t_{i-1}, t_{i}\right]}=\tau_{U}^{i}$. We also define the mesh size functions $h_{\tau}(\cdot)$ and $h_{\tau_{U}}(\cdot)$ by $\left.h_{\tau}(t)\right|_{t \in\left(t_{i-1}, t_{i}\right]}=h_{\tau^{i}}$ and $\left.h_{\tau_{U}}(t)\right|_{t \in\left(t_{i-1}, t_{i}\right]}=h_{\tau_{U}^{i}}$. In the following, for convenience, we use $\tau, \tau_{U}, h_{\tau}$, and $h_{\tau_{U}}$ to denote $\tau(t), \tau_{U}(t), h_{\tau}(t)$, and $h_{\tau_{U}}(t)$, respectively. Let $K_{i}^{h} \subseteq U_{i}^{h} \cap K$.

In order to present the full-discrete FEM approximation of $(O C P)^{h}$, we set $t=t_{i}$ in the first formula of (3.2), and in order to approximate derivative in time, we adopt the 1-order backward difference quotient. We also use the left rectangular quadrature formula to numerically approximate the integration.

Then the full-discrete FEM approximation form $(O C P)^{h k}$ of $(O C P)^{h}$ is as follows:

$$
\min _{u_{h}^{i} \in K_{i}^{h}} \frac{1}{2} \sum_{i=1}^{N} k_{i}\left\{\left\|y_{h}^{i}-z_{d}\right\|_{0, \Omega}^{2}+\alpha\left\|u_{h}^{i}\right\|_{0, \Omega}^{2}\right\}
$$

subject to

$$
\left\{\begin{array}{l}
\left(\frac{y_{h}^{i}-y_{h}^{i-1}}{k_{i}}, w_{h}\right)+a\left(\frac{y_{h}^{i}-y_{h}^{i-1}}{k_{i}}, w_{h}\right)+d\left(y_{h}^{i}, w_{h}\right)+\sum_{m=1}^{i} k_{m} c\left(t_{i}, t_{m-1} ; y_{h}^{m-1}, w_{h}\right) \\
=\left(f\left(x, t_{i}\right)+u_{h}^{i}, w_{h}\right), \quad \forall w_{h} \in V_{i}^{h}, \\
y_{h}^{0}=y_{0}^{h}(x), \quad x \in \Omega,
\end{array}\right.
$$

where $\left(y_{h}^{i}, u_{h}^{i}\right) \in V_{i}^{h} \times K_{i}^{h}(i=1,2, \ldots, N), u_{h}^{i}=u_{h}\left(t_{i}\right), y_{h}^{i}=y_{h}\left(t_{i}\right), i=1, \ldots, N$.

Following [3] and (2.4), we have that there exists a unique solution $\left(y_{h}^{i}, u_{h}^{i}\right) \in V_{i}^{h} \times K_{i}^{h}$ $(i=1,2, \ldots, N)$, and we know that a pair $\left(y_{h}^{i}, u_{h}^{i}\right)(i=1,2, \ldots, N)$ is a solution of $(O C P)^{h k}$ iff there is a costate $p_{h}^{i-1} \in V_{i-1}^{h}(i=1,2, \ldots, N)$ and the triplet $\left(y_{h}^{i}, p_{h}^{i-1}, u_{h}^{i}\right) \in V_{i}^{h} \times V_{i-1}^{h} \times K_{i}^{h}$ $(i=1,2, \ldots, N)$ satisfies the following optimality conditions $(O C P-O P T)^{h k}$ :

$$
\left\{\begin{array}{l}
\left(\frac{y_{h}^{i}-y_{h}^{i-1}}{k_{i}}, w_{h}\right)+a\left(\frac{y_{h}^{i}-y_{h}^{i-1}}{k_{i}}, w_{h}\right)+d\left(y_{h}^{i}, w_{h}\right)+\sum_{m=1}^{i} k_{m} c\left(t_{i}, t_{m-1} ; y_{h}^{m-1}, w_{h}\right) \\
\quad=\left(f\left(x, t_{i}\right)+u_{h}^{i}, w_{h}\right), \quad \forall w_{h} \in V_{i}^{h}, i=1, \ldots, N, \\
y_{h}^{0}=y_{0}^{h}(x), \quad x \in \Omega, \\
\left(\frac{p_{h}^{i-1}-p_{h}^{i}}{k_{i}}, q_{h}\right)+a\left(q_{h}, \frac{p_{h}^{i-1}-p_{h}^{i}}{k_{i}}\right)+d\left(q_{h}, p_{h}^{i-1}\right)+\sum_{m=i}^{N} k_{m} c\left(t_{m}, t_{i-1} ; q_{h}, p_{h}^{m}\right) \\
\quad=\left(y_{h}^{i}-y_{d}, q_{h}\right), \quad \forall q_{h} \in V_{i-1}^{h}, i=N, \ldots, 1, \\
p_{h}^{N}=0, \quad x \in \Omega, \\
\left(\alpha u_{h}^{i}+p_{h}^{i-1}, v_{h}-u_{h}^{i}\right) \geq 0, \quad \forall v_{h} \in K_{i}^{h}, i=1,2, \ldots, N .
\end{array}\right.
$$

We extend $\left\{\left(y_{h}^{i}, p_{h}^{i}, u_{h}^{i}\right)\right\}_{i=1}^{N}$ on $[0, T]$ and define:

$$
\begin{aligned}
& \left.Y_{h}\right|_{\left(t_{i-1}, t_{i}\right]}=\left(\left(t_{i}-t\right) y_{h}^{i-1}+\left(t-t_{i-1}\right) y_{h}^{i}\right) / k_{i}, \\
& \left.P_{h}\right|_{\left(t_{i-1}, t_{i}\right]}=\left(\left(t_{i}-t\right) p_{h}^{i-1}+\left(t-t_{i-1}\right) p_{h}^{i}\right) / k_{i}, \\
& \left.U_{h}\right|_{\left(t_{i-1}, t_{i}\right]}=u_{h}^{i} .
\end{aligned}
$$


For any function $w \in C\left(0, T ; L^{2}(\Omega)\right)$, let $\left.\hat{\omega}(x, t)\right|_{t \in\left(t_{i-1}, t_{i}\right]}=\omega\left(x, t_{i}\right),\left.\tilde{\omega}(x, t)\right|_{t \in\left(t_{i-1}, t_{i}\right]}=\omega\left(x, t_{i-1}\right)$, and $Y_{h}^{i}=Y_{h}\left(t_{i}\right), P_{h}^{i}=P_{h}\left(t_{i}\right), i=1, \ldots, N$.

Therefore, we can restate the full-discrete FEM optimality conditions (3.5) as follows:

$$
\left\{\begin{array}{l}
\left(\frac{\partial}{\partial t} Y_{h}, w_{h}\right)+a\left(\frac{\partial}{\partial t} Y_{h}, w_{h}\right)+d\left(\hat{Y}_{h}, w_{h}\right)+\tilde{g}\left(Y_{h}, w_{h}\right) \\
\quad=\left(\hat{f}+U_{h}, w_{h}\right), \quad \forall w_{h} \in V_{i}^{h}, i=1, \ldots, N, \\
Y_{h}(x, 0)=y_{0}^{h}(x), \quad x \in \Omega, \\
-\left(\frac{\partial}{\partial t} P_{h}, q_{h}\right)-a\left(q_{h}, \frac{\partial}{\partial t} P_{h}\right)+d\left(q_{h}, \tilde{P_{h}}\right)+\hat{h}\left(q_{h}, P_{h}\right) \\
\quad=\left(\hat{Y}_{h}-z_{d}, q_{h}\right), \quad \forall q_{h} \in V_{i-1}^{h}, i=N, \ldots, 1, \\
P_{h}(x, T)=0, \quad x \in \Omega, \\
\left(\alpha U_{h}+\tilde{P}_{h}, v_{h}-U_{h}\right) \geq 0, \quad U_{h} \in K_{i}^{h}, \forall v_{h} \in K_{i}^{h}, i=1,2, \ldots, N,
\end{array}\right.
$$

where

$$
\tilde{g}\left(Y_{h}, w_{h}\right)=\sum_{m=1}^{i} k_{m} c\left(t_{i}, t_{m-1} ; Y_{h}^{m-1}, w_{h}\right), \quad \hat{h}\left(q_{h}, P_{h}\right)=\sum_{m=i}^{N} k_{m} c\left(t_{m}, t_{i-1} ; q_{h}, P_{h}^{m}\right) .
$$

From [23] we have the following conclusion.

Theorem 3.1 Suppose that $P_{h}$ is known. Then we have the following solution for the variational inequality in (3.7):

$$
U_{h}=\frac{1}{\alpha} \Pi_{h}\left(-P_{h}+\max \left\{0, \frac{\int_{\Omega} P_{h}}{\int_{\Omega} 1}\right\}\right)
$$

where $\Pi_{h}: L^{2}$-projection $\left(L^{2}(\Omega) \rightarrow U^{h}\right)$.

\section{The upper and lower bounds of the a posteriori error estimates}

Since there is huge computational work in computing $(O C P-O P T)^{h k}$, we need to develop a highly efficient algorithm to compute $(O C P-O P T)^{h k}$. Here different partitions of meshes are used for the state and control.

First, we present the following two lemmas. Lemma 4.1 can be found in [24], and Lemma 4.2 can be found, for example, in Chapter 3 of [25].

Lemma 4.1 [24] Let $\pi_{h}$ be the average interpolation operator defined in [24]. Then, for $m=0$ or $1,1 \leq q \leq \infty$, and $v \in W^{1, q}\left(\Omega^{h}\right)$,

$$
\left|v-\pi_{h} v\right|_{m, q, \tau} \leq \sum_{\bar{\tau}^{\prime} \cap \bar{\tau} \neq \emptyset} C h_{\tau}^{1-m}|v|_{1, q, \tau^{\prime}}
$$

Lemma 4.2 [25] For all $v \in W^{1, q}(\Omega), 1 \leq q<\infty$,

$$
\|v\|_{0, q, \partial \tau} \leq C\left(h_{\tau}^{-\frac{1}{q}}\|v\|_{0, q, \tau}+h_{\tau}^{1-\frac{1}{q}}|v|_{1, q, \tau}\right)
$$




\subsection{Upper bound estimates}

To obtain upper bound estimates, we need to introduce the following auxiliary system:

$$
\left\{\begin{array}{l}
\left(\frac{\partial}{\partial t} y\left(U_{h}\right), w\right)+a\left(\frac{\partial}{\partial t} y\left(U_{h}\right), w\right)+d\left(y\left(U_{h}\right), w\right)+\int_{0}^{t} c\left(t, \tau ; y\left(U_{h}\right)(\tau), w\right) d \tau \\
\quad=\left(f+U_{h}, w\right), \quad \forall w \in H_{0}^{1}(\Omega), \\
y\left(U_{h}\right)(0)=y_{0}(x), \quad x \in \Omega, \\
-\left(q, \frac{\partial}{\partial t} p\left(U_{h}\right)\right)-a\left(q, \frac{\partial}{\partial t} p\left(U_{h}\right)\right)+d\left(q, p\left(U_{h}\right)\right)+\int_{t}^{T} c\left(\tau, t ; q, p\left(U_{h}\right)(\tau)\right) d \tau \\
\quad=\left(y\left(U_{h}\right)-z_{d}, q\right), \quad \forall q \in H_{0}^{1}(\Omega), \\
p\left(U_{h}\right)(T)=0, \quad x \in \Omega .
\end{array}\right.
$$

Lemma 4.3 Suppose that $(y, p, u)$ and $\left(Y_{h}, P_{h}, U_{h}\right)$ are the solutions of (2.4) and (3.7), respectively. Then we have

$$
\left\|u-U_{h}\right\|_{L^{2}\left(0, T ; L^{2}(\Omega)\right)}^{2} \leq C\left\{\eta_{1}^{2}+\left\|P_{h}-p\left(U_{h}\right)\right\|_{L^{2}\left(0, T ; L^{2}(\Omega)\right)}^{2}\right\},
$$

where $\eta_{1}^{2}$ is defined as follows:

$$
\eta_{1}^{2}=\sum_{i=1}^{N} \int_{t_{i-1}}^{t_{i}}\left\{\sum_{\tau_{U}} \int_{\tau_{U}}\left(P_{h}-\Pi_{h} P_{h}\right)^{2}\right\} d t .
$$

Proof First, we have

$$
\begin{aligned}
\alpha\left\|u-U_{h}\right\|_{L^{2}\left(0, T ; L^{2}(\Omega)\right)}^{2} \leq & \inf _{v_{h}(t) \in K^{h}} \int_{0}^{T}\left(P_{h}+\alpha U_{h}, v_{h}-u\right) d t \\
& +\int_{0}^{T}\left(P_{h}-p\left(U_{h}\right), u-U_{h}\right) d t+\int_{0}^{T}\left(p\left(U_{h}\right)-p, u-U_{h}\right) d t \\
= & I_{1}+I_{2}+I_{3} .
\end{aligned}
$$

Letting $v_{h}=\Pi_{h} u \in U^{h}$ in $I_{1}$, we have

$$
\begin{aligned}
& I_{1} \leq C(\delta) \sum_{i=1}^{N} \int_{t_{i-1}}^{t_{i}}\left\{\sum_{\tau_{U}} \int_{\tau_{U}}\left(-\Pi_{h} P_{h}+P_{h}\right)^{2}\right\} d t+\delta\left\|u-U_{h}\right\|_{L^{2}\left(0, T ; L^{2}(\Omega)\right)}^{2}, \\
& I_{2} \leq C\left\|P_{h}-p\left(U_{h}\right)\right\|_{L^{2}\left(0, T ; L^{2}(\Omega)\right)}^{2}+\delta\left\|u-U_{h}\right\|_{L^{2}\left(0, T ; L^{2}(\Omega)\right)^{\circ}}^{2}
\end{aligned}
$$

From (4.3) and (2.4), for any $t \in(0, T]$, we have

$$
\begin{aligned}
& \left(\frac{\partial}{\partial t}\left(y-y\left(U_{h}\right)\right), w\right)+a\left(\frac{\partial}{\partial t}\left(y-y\left(U_{h}\right)\right), w\right)+d\left(y-y\left(U_{h}\right), w\right) \\
& \quad+\int_{0}^{t} c\left(t, \tau ;\left(y-y\left(U_{h}\right)\right)(\tau), w\right) d \tau=\left(u-U_{h}, w\right), \quad \forall w \in V=H_{0}^{1}(\Omega),
\end{aligned}
$$

and

$$
\begin{aligned}
& -\left(q, \frac{\partial}{\partial t}\left(p-p\left(U_{h}\right)\right)\right)-a\left(q, \frac{\partial}{\partial t}\left(p-p\left(U_{h}\right)\right)\right)+d\left(q, p-p\left(U_{h}\right)\right) \\
& \quad+\int_{t}^{T} c\left(\tau, t ; q,\left(p-p\left(U_{h}\right)\right)(\tau)\right) d \tau=\left(y-y\left(U_{h}\right), q\right), \quad \forall q \in H_{0}^{1}(\Omega) .
\end{aligned}
$$


Chen Boundary Value Problems ( 2016) 2016:120

Page 8 of 25

From (4.8) and (4.9) we obtain

$$
\begin{aligned}
I_{3}= & \int_{0}^{T}\left(p\left(U_{h}\right)-p, u-U_{h}\right) d t \\
= & \int_{0}^{T}\left\{\left(\frac{\partial}{\partial t}\left(y-y\left(U_{h}\right)\right), p\left(U_{h}\right)-p\right)+a\left(\frac{\partial}{\partial t}\left(y-y\left(U_{h}\right)\right), p\left(U_{h}\right)-p\right)\right. \\
& \left.+d\left(y-y\left(U_{h}\right), p\left(U_{h}\right)-p\right)+\int_{0}^{t} c\left(t, \tau ;\left(y-y\left(U_{h}\right)\right)(\tau),\left(p\left(U_{h}\right)-p\right)(t)\right) d \tau\right\} d t \\
= & \int_{0}^{T}\left\{-\left(y-y\left(U_{h}\right), \frac{\partial}{\partial t}\left(p\left(U_{h}\right)-p\right)\right)-a\left(y-y\left(U_{h}\right), \frac{\partial}{\partial t}\left(p\left(U_{h}\right)-p\right)\right)\right. \\
& \left.+d\left(y-y\left(U_{h}\right), p\left(U_{h}\right)-p\right)+\int_{t}^{T} c\left(\tau, t ;\left(y-y\left(U_{h}\right)\right)(t),\left(p\left(U_{h}\right)-p\right)(\tau)\right) d \tau\right\} d t \\
= & \int_{0}^{T}-\left(y-y\left(U_{h}\right), y-y\left(U_{h}\right)\right) d t \leq 0 .
\end{aligned}
$$

We can obtain (4.4) from (4.6)-(4.10). This completes the proof.

Since $y-Y_{h}=y-y\left(U_{h}\right)+y\left(U_{h}\right)-Y_{h}, p-P_{h}=p-p\left(U_{h}\right)+p\left(U_{h}\right)-P_{h}$, we now first present the estimates of $Y_{h}-y\left(U_{h}\right)$ and $P_{h}-p\left(U_{h}\right)$.

Lemma 4.4 Suppose that $\left(Y_{h}, P_{h}, U_{h}\right)$ and $\left(y\left(U_{h}\right), p\left(U_{h}\right)\right)$ are the solutions of (3.7) and (4.3), respectively. Then we have

$$
\begin{aligned}
& \left\|Y_{h}-y\left(U_{h}\right)\right\|_{L^{\infty}\left(0, T ; L^{2}(\Omega)\right)}^{2}+\left\|Y_{h}-y\left(U_{h}\right)\right\|_{L^{2}\left(0, T ; H^{1}(\Omega)\right)}^{2} \\
& +\left\|\frac{\partial}{\partial t}\left(Y_{h}-y\left(U_{h}\right)\right)\right\|_{L^{2}\left(0, T ; H^{1}(\Omega)\right)}^{2}+\left\|P_{h}-p\left(U_{h}\right)\right\|_{L^{\infty}\left(0, T ; L^{2}(\Omega)\right)}^{2} \\
& \quad+\left\|P_{h}-p\left(U_{h}\right)\right\|_{L^{2}\left(0, T ; H^{1}(\Omega)\right)}^{2}+\left\|\frac{\partial}{\partial t}\left(P_{h}-p\left(U_{h}\right)\right)\right\|_{L^{2}\left(0, T ; H^{1}(\Omega)\right)}^{2} \leq C \sum_{i=2}^{13} \eta_{i}^{2},
\end{aligned}
$$

where $\eta_{i}(i=2, \ldots, 13)$ is defined as follows:

$$
\begin{aligned}
\eta_{2}^{2}= & \sum_{i=1}^{N} k_{i}\left\{\sum _ { \tau } h _ { \tau } ^ { 2 } \int _ { \tau } \left(\hat{Y}_{h}-z_{d}+\frac{\partial}{\partial t} P_{h}-\operatorname{div}\left(A^{*} \nabla \frac{\partial}{\partial t} P_{h}\right)+\operatorname{div}\left(D^{*} \nabla \tilde{P}_{h}\right)\right.\right. \\
& \left.+\sum_{m=i}^{N} k_{m} \operatorname{div}\left(C^{*}\left(t_{m}, t_{i-1}\right) \nabla P_{h}^{m}\right)\right)^{2}+\sum_{l} h_{l} \int_{l}\left[\left(A^{*} \nabla \frac{\partial}{\partial t} P_{h}\right) \cdot n-\left(D^{*} \nabla \tilde{P}_{h}\right) \cdot n\right. \\
& \left.\left.-\sum_{m=i}^{N} k_{m}\left(C^{*}\left(t_{m}, t_{i-1}\right) \nabla P_{h}^{m}\right) \cdot n\right]^{2}\right\} \\
\eta_{3}^{2}= & \sum_{i=1}^{N} \frac{k_{i}}{3}\left|P_{h}^{i-1}-P_{h}^{i}\right|_{1, \Omega}^{2}, \\
\eta_{4}^{2}= & \sum_{i=1}^{N} \int_{t_{i-1}}^{t_{i}}\left(\sum_{m=i}^{N} \int_{t_{m-1}}^{t_{m}}\left\|\left(C^{*}\left(t_{m}, t_{i-1}\right)-C^{*}(\tau, t)\right) \nabla P_{h}^{m}\right\|_{0, \Omega}^{2} d \tau\right) d t,
\end{aligned}
$$




$$
\begin{aligned}
\eta_{5}^{2}= & \sum_{i=1}^{N} k_{i} \sum_{m=i}^{N} \frac{k_{m}}{3}\left|P_{h}^{m-1}-P_{h}^{m}\right|_{1, \Omega}^{2} \\
\eta_{6}^{2}= & \sum_{i=1}^{N} k_{i}^{3}\left|\tilde{P}_{h}\right|_{1, \Omega}^{2}, \\
\eta_{7}^{2}= & \sum_{i=1}^{N} k_{i}\left\{\sum _ { \tau } h _ { \tau } ^ { 2 } \int _ { \tau } \left(\hat{f}+U_{h}-\frac{\partial}{\partial t} Y_{h}+\operatorname{div}\left(A \nabla \frac{\partial}{\partial t} Y_{h}\right)\right.\right. \\
& \left.+\operatorname{div}\left(D \nabla \hat{Y}_{h}\right)+\sum_{m=1}^{i} k_{m} \operatorname{div}\left(C\left(t_{i}, t_{m-1}\right) \nabla Y_{h}^{m-1}\right)\right)^{2}+\sum_{l} h_{l} \iint_{l}\left[\left(A \nabla \frac{\partial}{\partial t} Y_{h}\right) \cdot n\right. \\
& \left.\left.+\left(D \nabla \hat{Y}_{h}\right) \cdot n+\sum_{m=1}^{i} k_{m}\left(C\left(t_{i}, t_{m-1}\right) \nabla Y_{h}^{m-1}\right) \cdot n\right]^{2}\right\} \\
\eta_{8}^{2}= & \|f-\hat{f}\|_{L^{2}\left(0, T ; L^{2}(\Omega)\right)^{\prime}}^{2} \\
\eta_{9}^{2}= & \sum_{i=1}^{N} \frac{k_{i}}{3}\left|Y_{h}^{i-1}-Y_{h}^{i}\right|_{1, \Omega}^{2}, \\
\eta_{10}^{2}= & \sum_{i=1}^{N} \int_{t_{i-1}}^{t_{i}}\left(\sum_{m=1}^{i} \int_{t_{m-1}}^{t_{m}}\left\|\left(C\left(t_{i}, t_{m-1}\right)-C(t, \tau)\right) \nabla Y_{h}^{m-1}\right\|_{0, \Omega}^{2} d \tau\right) d t \\
\eta_{11}^{2}= & \sum_{i=1}^{N} k_{i} \sum_{m=1}^{i} \frac{k_{m}}{3}\left|Y_{h}^{m-1}-Y_{h}^{m}\right|_{1, \Omega}^{2}, \\
\eta_{12}^{2}= & \sum_{i=1}^{N} k_{i}^{3}\left|\hat{Y}_{h}\right|_{1, \Omega}^{2}, \\
\eta_{13}^{2}= & \left\|Y_{h}(x, 0)-y_{0}(x)\right\|_{0, \Omega}^{2},
\end{aligned}
$$

where $l$ is a face of an element $\tau, h_{l}$ is the maximal diameter of $l$, and we denote the normal derivative jumps over the interior face $l$ by $\left[\nabla \tilde{P}_{h} \cdot n\right]$ and $\left[\nabla \hat{Y}_{h} \cdot n\right]$ as follows:

$$
\begin{aligned}
& {\left[\nabla \tilde{P}_{h} \cdot n\right]_{l}=\left(\left.\nabla \tilde{P}_{h}\right|_{\tau_{l}^{1}}-\left.\nabla \tilde{P}_{h}\right|_{\tau_{l}^{2}}\right) \cdot n,} \\
& {\left[\nabla \hat{Y}_{h} \cdot n\right]_{l}=\left(\left.\nabla \hat{Y}_{h}\right|_{\tau_{l}^{1}}-\left.\nabla \hat{Y}_{h}\right|_{\tau_{l}^{2}}\right) \cdot n,}
\end{aligned}
$$

where $n$ is the unit normal vector on $l=\tau_{l}^{1} \cap \tau_{l}^{2}$. For simplicity, we suppose that when $l \subset \partial \Omega$, $\left[\nabla \tilde{P}_{h} \cdot n\right]_{l}=0$ and $\left[\nabla \hat{Y}_{h} \cdot n\right]_{l}=0$.

Proof First, we estimate $P_{h}-p\left(U_{h}\right)$. Suppose that the average interpolation operator $\pi_{h}$ is defined as in [24] and define

$$
\begin{aligned}
\left\langle R\left(U_{h}\right), v\right\rangle= & -\left(\frac{\partial}{\partial t}\left(p\left(U_{h}\right)-P_{h}\right), v\right)-a\left(v, \frac{\partial}{\partial t}\left(p\left(U_{h}\right)-P_{h}\right)\right)+d\left(v, p\left(U_{h}\right)-P_{h}\right) \\
& +\int_{t}^{T} c\left(\tau, t ; v, p\left(U_{h}\right)(\tau)\right) d \tau-\hat{h}\left(v, P_{h}\right) .
\end{aligned}
$$


Then, letting $q=\pi_{h} v$ in (4.3) and $q_{h}=\pi_{h} v$ in (3.7), respectively, then subtracting (3.7) from (4.3), for all $t \in\left[t_{i-1}, t_{i}\right]$, we have

$$
\begin{gathered}
-\left(\pi_{h} v, \frac{\partial}{\partial t}\left(p\left(U_{h}\right)-P_{h}\right)\right)-a\left(\pi_{h} v, \frac{\partial}{\partial t}\left(p\left(U_{h}\right)-P_{h}\right)\right)+d\left(\pi_{h} v, p\left(U_{h}\right)-\tilde{P}_{h}\right) \\
+\int_{t}^{T} c\left(\tau, t ; \pi_{h} v, p\left(U_{h}\right)(\tau)\right) d \tau-\hat{h}\left(\pi_{h} v, P_{h}\right)=\left(y\left(U_{h}\right)-\hat{Y}_{h}, \pi_{h} v\right) .
\end{gathered}
$$

So we have

$$
\begin{aligned}
& -\left(\pi_{h} v, \frac{\partial}{\partial t}\left(p\left(U_{h}\right)-P_{h}\right)\right)-a\left(\pi_{h} v, \frac{\partial}{\partial t}\left(p\left(U_{h}\right)-P_{h}\right)\right)+d\left(\pi_{h} v, p\left(U_{h}\right)-P_{h}\right) \\
& \quad+\int_{t}^{T} c\left(\tau, t ; \pi_{h} v, p\left(U_{h}\right)(\tau)\right) d \tau-\hat{h}\left(\pi_{h} v, P_{h}\right) \\
& =\left(y\left(U_{h}\right)-\hat{Y}_{h}, \pi_{h} v\right)+d\left(\pi_{h} v, \tilde{P}_{h}-P_{h}\right) .
\end{aligned}
$$

Then from (4.14) and (4.3) we obtain

$$
\begin{aligned}
-\left(\frac{\partial}{\partial t}\right. & \left.\left(p\left(U_{h}\right)-P_{h}\right), v\right)-a\left(v, \frac{\partial}{\partial t}\left(p\left(U_{h}\right)-P_{h}\right)\right)+d\left(v, p\left(U_{h}\right)-P_{h}\right) \\
& +\int_{t}^{T} c\left(\tau, t ; v, p\left(U_{h}\right)(\tau)\right) d \tau-\hat{h}\left(v, P_{h}\right) \\
= & -\left(\frac{\partial}{\partial t}\left(p\left(U_{h}\right)-P_{h}\right), v-\pi_{h} v\right)-a\left(v-\pi_{h} v, \frac{\partial}{\partial t}\left(p\left(U_{h}\right)-P_{h}\right)\right) \\
& +d\left(v-\pi_{h} v, p\left(U_{h}\right)-P_{h}\right)+\int_{t}^{T} c\left(\tau, t ; v-\pi_{h} v, p\left(U_{h}\right)(\tau)\right) d \tau-\hat{h}\left(v-\pi_{h} v, P_{h}\right) \\
& -\left(\frac{\partial}{\partial t}\left(p\left(U_{h}\right)-P_{h}\right), \pi_{h} v\right)-a\left(\pi_{h} v, \frac{\partial}{\partial t}\left(p\left(U_{h}\right)-P_{h}\right)\right)+d\left(\pi_{h} v, p\left(U_{h}\right)-P_{h}\right) \\
& +\int_{t}^{T} c\left(\tau, t ; \pi_{h} v, p\left(U_{h}\right)(\tau)\right) d \tau-\hat{h}\left(\pi_{h} v, P_{h}\right) \\
= & -\left(\frac{\partial}{\partial t}\left(p\left(U_{h}\right)-P_{h}\right), v-\pi_{h} v\right)-a\left(v-\pi_{h} v, \frac{\partial}{\partial t}\left(p\left(U_{h}\right)-P_{h}\right)\right) \\
& +d\left(v-\pi_{h} v, p\left(U_{h}\right)-P_{h}\right)+\int_{t}^{T} c\left(\tau, t ; v-\pi_{h} v, p\left(U_{h}\right)(\tau)\right) d \tau-\hat{h}\left(v-\pi_{h} v, P_{h}\right) \\
& +\left(y\left(U_{h}\right)-\hat{Y}_{h}, \pi_{h} v\right)+d\left(\pi_{h} v, \tilde{P}_{h}-P_{h}\right) \\
= & -\left(\frac{\partial}{\partial t} p\left(U_{h}\right), v-\pi_{h} v\right)-a\left(v-\pi_{h} v, \frac{\partial}{\partial t} p\left(U_{h}\right)\right)+d\left(v-\pi_{h} v, p\left(U_{h}\right)\right) \\
& +\left(\frac{\partial}{\partial t} P_{h}, v-\pi_{h} v\right)+a\left(v-\pi_{h} v, \frac{\partial}{\partial t} P_{h}\right)-d\left(v-\pi_{h} v, P_{h}\right)-\hat{h}\left(v-\pi_{h} v, P_{h}\right) \\
& +\int_{t}^{T} c\left(\tau, t ; v-\pi_{h} v, p\left(U_{h}\right)(\tau)\right) d \tau+\left(\frac{\partial}{\partial t} P_{h}, v-\pi_{h} v\right)+a\left(v-\pi_{h} v, \frac{\partial}{\partial t} P_{h}\right) \\
& -d\left(v-\pi_{h} v, P_{h}\right)-\hat{h}\left(v-\pi_{h} v, P_{h}\right)+\left(y\left(U_{h}\right)-\hat{Y}_{h}, \pi_{h} v\right)+d\left(\pi_{h} v, \tilde{P}_{h}-P_{h}\right) \\
& \left(U_{h}, v-\pi_{h} v\right)+\left(y\left(U_{h}\right)-\hat{Y}_{h}, \pi_{h} v\right)+d\left(\pi_{h} v, \tilde{P}_{h}-P_{h}\right) \\
&
\end{aligned}
$$




$$
\begin{aligned}
= & \left(y\left(U_{h}\right)-\hat{Y}_{h}+\hat{Y}_{h}-z_{d}, v-\pi_{h} v\right)+\left(y\left(U_{h}\right)-\hat{Y}_{h}, \pi_{h} v\right)+d\left(\pi_{h} v, \tilde{P}_{h}-P_{h}\right) \\
& +\left(\frac{\partial}{\partial t} P_{h}, v-\pi_{h} v\right)+a\left(v-\pi_{h} v, \frac{\partial}{\partial t} P_{h}\right)-d\left(v-\pi_{h} v, P_{h}\right)-\hat{h}\left(v-\pi_{h} v, P_{h}\right) \\
= & \left(y\left(U_{h}\right)-\hat{Y}_{h}, v\right)+\left(\hat{Y}_{h}-z_{d}, v-\pi_{h} v\right)+\left(\frac{\partial}{\partial t} P_{h}, v-\pi_{h} v\right)+a\left(v-\pi_{h} v, \frac{\partial}{\partial t} P_{h}\right) \\
& -d\left(v-\pi_{h} v, \tilde{P}_{h}\right)+d\left(v, \tilde{P}_{h}-P_{h}\right)-\hat{h}\left(v-\pi_{h} v, P_{h}\right) \\
= & \left(y\left(U_{h}\right)-\hat{Y}_{h}, v\right)+d\left(v, \tilde{P}_{h}-P_{h}\right)+\left(\hat{Y}_{h}-z_{d}, v-\pi_{h} v\right)+\left(\frac{\partial}{\partial t} P_{h}, v-\pi_{h} v\right) \\
& +a\left(v-\pi_{h} v, \frac{\partial}{\partial t} P_{h}\right)-d\left(v-\pi_{h} v, \tilde{P}_{h}\right)-\hat{h}\left(v-\pi_{h} v, P_{h}\right) .
\end{aligned}
$$

From the definition of $a, d, \hat{h}$, the Green formula, and (4.15) we have

$$
\begin{aligned}
& -\left(\frac{\partial}{\partial t}\left(p\left(U_{h}\right)-P_{h}\right), v\right)-a\left(v, \frac{\partial}{\partial t}\left(p\left(U_{h}\right)-P_{h}\right)\right)+d\left(v, p\left(U_{h}\right)-P_{h}\right) \\
& \quad+\int_{t}^{T} c\left(\tau, t ; v, p\left(U_{h}\right)(\tau)\right) d \tau-\hat{h}\left(v, P_{h}\right) \\
& =\left(y\left(U_{h}\right)-\hat{Y}_{h}, v\right)+d\left(v, \tilde{P}_{h}-P_{h}\right)+\sum_{\tau} \int_{\tau}\left(\hat{Y}_{h}-z_{d}+\frac{\partial}{\partial t} P_{h}-\operatorname{div}\left(A^{*} \nabla \frac{\partial}{\partial t} P_{h}\right)\right. \\
& \left.\quad+\operatorname{div}\left(D^{*} \nabla \tilde{P}_{h}\right)+\sum_{m=i}^{N} k_{m} \operatorname{div}\left(C^{*}\left(t_{m}, t_{i-1}\right) \nabla P_{h}^{m}\right)\right)\left(v-\pi_{h} v\right) \\
& \quad+\sum_{l} \int_{l}\left[\left(A^{*} \nabla \frac{\partial P_{h}}{\partial t}\right) \cdot n-\left(D^{*} \nabla \tilde{P}_{h}\right) \cdot n-\sum_{m=i}^{N} k_{m}\left(C^{*}\left(t_{m}, t_{i-1}\right) \nabla P_{h}^{m}\right) \cdot n\right]\left(v-\pi_{h} v\right) \\
& =I_{1}+\cdots+I_{4} .
\end{aligned}
$$

Let

$$
G=-\left(\frac{\partial}{\partial t}\left(p\left(U_{h}\right)-P_{h}\right), v\right)-a\left(v, \frac{\partial}{\partial t}\left(p\left(U_{h}\right)-P_{h}\right)\right)+d\left(v, p\left(U_{h}\right)-P_{h}\right) .
$$

Then we have

$$
G=I_{1}+\cdots+I_{4}+\hat{h}\left(v, P_{h}\right)-\int_{t}^{T} c\left(\tau, t ; v, p\left(U_{h}\right)(\tau)\right) d \tau,
$$

where, for all $t \in\left[t_{i-1}, t_{i}\right]$,

$$
\begin{aligned}
J= & \hat{h}\left(v, P_{h}\right)-\int_{t}^{T} c\left(\tau, t ; v, p\left(U_{h}\right)(\tau)\right) d \tau \\
= & \sum_{m=i}^{N} \int_{t_{m-1}}^{t_{m}}\left(\left(C\left(t_{m}, t_{i-1}\right)-C(\tau, t)\right) \nabla v, \nabla P_{h}^{m}\right) d \tau+\sum_{m=i}^{N} \int_{t_{m-1}}^{t_{m}} c\left(\tau, t ; v, P_{h}^{m}-P_{h}\right) d \tau \\
& +\sum_{m=i}^{N} \int_{t_{m-1}}^{t_{m}} c\left(\tau, t ; v,\left(P_{h}-P\left(U_{h}\right)\right)(\tau)\right) d \tau+\int_{t_{i-1}}^{t} c\left(\tau, t ; v, p\left(U_{h}\right)-P_{h}\right) d \tau
\end{aligned}
$$




$$
\begin{aligned}
& +\int_{t_{i-1}}^{t} c\left(\tau, t ; v, P_{h}-\tilde{P}_{h}\right) d \tau+\int_{t_{i-1}}^{t} c\left(\tau, t ; v, \tilde{P}_{h}\right) d \tau \\
= & J_{1}+\cdots+J_{6} .
\end{aligned}
$$

Then from Theorem 3.1, Lemma 4.1, and 4.2, setting $v=p\left(U_{h}\right)-P_{h}$ in (4.16)-(4.19), we have

$$
G \geq-\frac{1}{2} \frac{d}{d t}\left\|p\left(U_{h}\right)-P_{h}\right\|_{0, \Omega}^{2}-\frac{1}{2} \frac{d}{d t} a\left(p\left(U_{h}\right)-P_{h}, p\left(U_{h}\right)-P_{h}\right)+c\left\|p\left(U_{h}\right)-P_{h}\right\|_{1, \Omega}^{2} .
$$

Then from [23], for all $t \in\left[t_{i-1}, t_{i}\right]$, using

$$
\tilde{P}_{h}-P_{h}=\frac{t-t_{i-1}}{k_{i}}\left(P_{h}^{i-1}-P_{h}^{i}\right)
$$

we have the following estimates:

$$
\begin{aligned}
& I_{1}+I_{2} \leq\left\|y\left(U_{h}\right)-\hat{Y}_{h}\right\|_{0, \Omega}^{2}+C\left|\tilde{P}_{h}-P_{h}\right|_{1, \Omega}^{2}+\varepsilon\left\|p\left(U_{h}\right)-P_{h}\right\|_{1, \Omega^{\prime}}^{2} \\
& I_{3}+I_{4} \leq C\left\{\sum _ { \tau } h _ { \tau } ^ { 2 } \int _ { \tau } \left(\hat{Y}_{h}-z_{d}+\frac{\partial}{\partial t} P_{h}-\operatorname{div}\left(A^{*} \nabla \frac{\partial}{\partial t} P_{h}\right)+\operatorname{div}\left(D^{*} \nabla \tilde{P}_{h}\right)\right.\right. \\
&\left.+\sum_{m=i}^{N} k_{m} \operatorname{div}\left(C^{*}\left(t_{m}, t_{i-1}\right) \nabla P_{h}^{m}\right)\right)^{2}+\sum_{l} h_{l} \int\left[\int _ { l } \left[\left(A^{*} \nabla \frac{\partial}{\partial t} P_{h}\right) \cdot n\right.\right. \\
&\left.\left.-\left(D^{*} \nabla \tilde{P}_{h}\right) \cdot n-\sum_{m=i}^{N} k_{m}\left(C^{*}\left(t_{m}, t_{i-1}\right) \nabla P_{h}^{m}\right) \cdot n\right]^{2}\right\}+\varepsilon\left\|p\left(U_{h}\right)-P_{h}\right\|_{1, \Omega}^{2} \\
& J_{1} \leq C \sum_{m=i}^{N} \int_{t_{m-1}}^{t_{m}}\left\|\left(C^{*}\left(t_{m}, t_{i-1}\right)-C^{*}(\tau, t)\right) \nabla P_{h}^{m}\right\|_{0, \Omega}^{2} d \tau+\varepsilon\left\|p\left(U_{h}\right)-P_{h}\right\|_{1, \Omega^{\prime}}^{2} \\
& J_{2} \leq C \sum_{m=i}^{N} \frac{k_{m}}{3}\left|P_{h}^{m-1}-P_{h}^{m}\right|_{1, \Omega}^{2}+\varepsilon\left\|p\left(U_{h}\right)-P_{h}\right\|_{1, \Omega^{\prime}}^{2} \\
& J_{3} \leq C \int_{t_{i-1}}^{T}\left\|\left(p\left(U_{h}\right)-P_{h}\right)(\tau)\right\|_{1, \Omega}^{2} d \tau+\varepsilon\left\|p\left(U_{h}\right)-P_{h}\right\|_{1, \Omega^{\prime}}^{2} \\
& J_{4} \leq C \int_{t_{i-1}}^{t}\left\|p\left(U_{h}\right)-P_{h}\right\|_{1, \Omega}^{2} d \tau+\varepsilon\left\|p\left(U_{h}\right)-P_{h}\right\|_{1, \Omega}^{2} \\
& J_{5} \leq C \int_{t_{i-1}}^{t_{i}}\left|P_{h}-\tilde{P}_{h}\right|_{1, \Omega}^{2} d \tau+\varepsilon\left\|p\left(U_{h}\right)-P_{h}\right\|_{1, \Omega}^{2} \\
& \leq C \frac{k_{i}}{3}\left|P_{h}^{i-1}-P_{h}^{i}\right|_{1, \Omega}^{2}+\varepsilon\left\|p\left(U_{h}\right)-P_{h}\right\|_{1, \Omega}^{2} \\
& J_{6} \leq C k_{i}^{2}\left|\tilde{P}_{h}\right|_{1, \Omega}^{2}+\varepsilon\left\|p\left(U_{h}\right)-P_{h}\right\|_{1, \Omega}^{2} .
\end{aligned}
$$

Setting $\varepsilon$ small enough, from (4.20)-(4.21) we obtain:

$$
\begin{aligned}
& -\frac{1}{2} \frac{d}{d t}\left\|p\left(U_{h}\right)-P_{h}\right\|_{0, \Omega}^{2}-\frac{1}{2} \frac{d}{d t} a\left(p\left(U_{h}\right)-P_{h}, p\left(U_{h}\right)-P_{h}\right)+c\left\|p\left(U_{h}\right)-P_{h}\right\|_{1, \Omega}^{2} \\
& \quad \leq C\left\{\left\|y\left(U_{h}\right)-\hat{Y}_{h}\right\|_{0, \Omega}^{2}+\left|\tilde{P}_{h}-P_{h}\right|_{1, \Omega}^{2}+\sum_{\tau} h_{\tau}^{2} \int_{\tau}\left(\hat{Y}_{h}-z_{d}+\frac{\partial}{\partial t} P_{h}\right.\right.
\end{aligned}
$$




$$
\begin{aligned}
& \left.-\operatorname{div}\left(A^{*} \nabla \frac{\partial}{\partial t} P_{h}\right)+\operatorname{div}\left(D^{*} \nabla \tilde{P}_{h}\right)+\sum_{m=i}^{N} k_{m} \operatorname{div}\left(C^{*}\left(t_{m}, t_{i-1}\right) \nabla P_{h}^{m}\right)\right)^{2} \\
& +\sum_{l} h_{l} \int_{l}\left[\left(A^{*} \nabla \frac{\partial}{\partial t} P_{h}\right) \cdot n-\left(D^{*} \nabla \tilde{P}_{h}\right) \cdot n-\sum_{m=i}^{N} k_{m}\left(C^{*}\left(t_{m}, t_{i-1}\right) \nabla P_{h}^{m}\right) \cdot n\right]^{2} \\
& +\sum_{m=i}^{N} \int_{t_{m-1}}^{t_{m}}\left\|\left(C^{*}\left(t_{m}, t_{i-1}\right)-C^{*}(\tau, t)\right) \nabla P_{h}^{m}\right\|_{0, \Omega}^{2} d \tau \\
& \left.+\sum_{m=i}^{N} \frac{k_{m}}{3}\left|P_{h}^{m-1}-P_{h}^{m}\right|_{1, \Omega}^{2}+\int_{t_{i-1}}^{T}\left\|p\left(U_{h}\right)-P_{h}\right\|_{1, \Omega}^{2} d \tau+k_{i}^{2}\left|\tilde{P}_{h}\right|_{1, \Omega}^{2}\right\} .
\end{aligned}
$$

Then integrating (4.22) from 0 to $T$ in time, we have

$$
\begin{aligned}
&\left\|p\left(U_{h}\right)-P_{h}\right\|_{L^{\infty}\left(0, T ; L^{2}(\Omega)\right)}^{2}+\left\|p\left(U_{h}\right)-P_{h}\right\|_{L^{2}\left(0, T ; H^{1}(\Omega)\right)}^{2} \\
& \leq C\left\{\sum _ { i = 1 } ^ { N } k _ { i } \left(\sum _ { \tau } h _ { \tau } ^ { 2 } \int _ { \tau } \left(\hat{Y}_{h}-z_{d}+\frac{\partial}{\partial t} P_{h}-\operatorname{div}\left(A^{*} \nabla \frac{\partial}{\partial t} P_{h}\right)+\operatorname{div}\left(D^{*} \nabla \tilde{P}_{h}\right)\right.\right.\right. \\
&\left.+\sum_{m=i}^{N} k_{m} \operatorname{div}\left(C^{*}\left(t_{m}, t_{i-1}\right) \nabla P_{h}^{m}\right)\right)^{2}+\sum_{l} h_{l} \int\left[\int _ { l } \left[\left(A^{*} \nabla \frac{\partial}{\partial t} P_{h}\right) \cdot n-\left(D^{*} \nabla \tilde{P}_{h}\right) \cdot n\right.\right. \\
&\left.\left.-\sum_{m=i}^{N} k_{m}\left(C^{*}\left(t_{m}, t_{i-1}\right) \nabla P_{h}^{m}\right) \cdot n\right]^{2}\right)+\sum_{i=1}^{N} \frac{k_{i}}{3}\left|P_{h}^{i-1}-P_{h}^{i}\right|_{1, \Omega}^{2}+\sum_{i=1}^{N} k_{i}^{3}\left|\tilde{P}_{h}\right|_{1, \Omega}^{2} \\
&+\sum_{i=1}^{N} \int_{t_{i-1}}^{t_{i}}\left(\sum_{m=i}^{N} \int_{t_{m-1}}^{t_{m}}\left\|\left(C^{*}\left(t_{m}, t_{i-1}\right)-C^{*}(\tau, t)\right) \nabla P_{h}^{m}\right\|_{0, \Omega}^{2} d \tau\right) d t \\
&\left.+\sum_{i=1}^{N} k_{i} \sum_{m=i}^{N} \frac{k_{m}}{3}\left|P_{h}^{m-1}-P_{h}^{m}\right|_{1, \Omega}^{2}+\sum_{i=1}^{N}\left\|y\left(U_{h}\right)-\hat{Y}_{h}\right\|_{L^{2}\left(t_{i-1}, t_{i} L^{2}(\Omega)\right)}^{2}\right\} \\
&= C\left(\eta_{2}^{2}+\cdots+\eta_{6}^{2}+\sum_{i=1}^{N}\left\|y\left(U_{h}\right)-\hat{Y}_{h}\right\|_{L^{2}\left(t_{i-1}, t_{i j} L^{2}(\Omega)\right)}^{2}\right)
\end{aligned}
$$

In the same way, setting $v=\frac{\partial}{\partial t}\left(p\left(U_{h}\right)-P_{h}\right)$ in (4.16)-(4.19), we obtain:

$$
\left\|\frac{\partial}{\partial t}\left(p\left(U_{h}\right)-P_{h}\right)\right\|_{L^{2}\left(0, T ; H^{1}(\Omega)\right)}^{2} \leq C\left(\eta_{2}^{2}+\cdots+\eta_{6}^{2}+\sum_{i=1}^{N}\left\|y\left(U_{h}\right)-\hat{Y}_{h}\right\|_{L^{2}\left(t_{i-1}, t_{i} ; L^{2}(\Omega)\right)}^{2}\right)
$$

where

$$
\begin{aligned}
& \sum_{i=1}^{N}\left\|y\left(U_{h}\right)-\hat{Y}_{h}\right\|_{L^{2}\left(t_{i-1}, t_{i} L^{2}(\Omega)\right)}^{2} \\
& \quad \leq C\left(\sum_{i=1}^{N}\left\|y\left(U_{h}\right)-Y_{h}\right\|_{L^{2}\left(t_{i-1}, t_{i} L^{2}(\Omega)\right)}^{2}+\sum_{i=1}^{N}\left\|Y_{h}-\hat{Y}_{h}\right\|_{L^{2}\left(t_{i-1}, t_{i} ; L^{2}(\Omega)\right)}^{2}\right) \\
& \quad \leq C\left(\left\|y\left(U_{h}\right)-Y_{h}\right\|_{L^{2}\left(0, T ; L^{2}(\Omega)\right)}^{2}+\sum_{i=1}^{N} \frac{k_{i}}{3}\left|Y_{h}^{i-1}-Y_{h}^{i}\right|_{1, \Omega}^{2}\right) .
\end{aligned}
$$


Next, we estimate $y\left(U_{h}\right)-Y_{h}$. Let

$$
\begin{aligned}
\left\langle Q\left(U_{h}\right), v\right\rangle= & \left(\frac{\partial}{\partial t}\left(y\left(U_{h}\right)-Y_{h}\right), v\right)+a\left(\frac{\partial}{\partial t}\left(y\left(U_{h}\right)-Y_{h}\right), v\right)+d\left(y\left(U_{h}\right)-Y_{h}, v\right) \\
& +\int_{0}^{t} c\left(t, \tau ; y\left(U_{h}\right)(\tau), v\right) d \tau-\tilde{g}\left(Y_{h}, v\right) .
\end{aligned}
$$

We obviously have

$$
\begin{aligned}
& \left(\frac{\partial}{\partial t}\left(y\left(U_{h}\right)-Y_{h}\right), v\right)+a\left(\frac{\partial}{\partial t}\left(y\left(U_{h}\right)-Y_{h}\right), v\right)+d\left(y\left(U_{h}\right)-Y_{h}, v\right)-\tilde{g}\left(Y_{h}, v\right) \\
& \quad+\int_{0}^{t} c\left(t, \tau ; y\left(U_{h}\right)(\tau), v\right) d \tau \\
& =(f-\hat{f}, v)+d\left(\hat{Y}_{h}-Y_{h}, v\right)+\left\{\sum _ { \tau } \int _ { \tau } \left(\hat{f}+U_{h}-\frac{\partial}{\partial t} Y_{h}+\operatorname{div}\left(A \nabla \frac{\partial}{\partial t} Y_{h}\right)\right.\right. \\
& \left.\quad+\operatorname{div}\left(D \nabla \hat{Y}_{h}\right)+\sum_{m=1}^{i} k_{m} \operatorname{div}\left(C\left(t_{i}, t_{m-1}\right) \nabla Y_{h}^{m-1}\right)\right) \\
& \quad-\sum_{l} \int\left[\left(A \nabla \frac{\partial}{\partial t} Y_{h}\right) \cdot n+\left(D \nabla \hat{Y}_{h}\right) \cdot n\right. \\
& \left.\left.\quad+\sum_{m=1}^{i} k_{m}\left(C\left(t_{i}, t_{m-1}\right) \nabla Y_{h}^{m-1}\right) \cdot n\right]\right\}\left(v-\pi_{h} v\right) \\
& =L_{1}+L_{2}+L_{3} .
\end{aligned}
$$

Therefore,

$$
\begin{gathered}
\left(\frac{\partial}{\partial t}\left(y\left(U_{h}\right)-Y_{h}\right), v\right)+a\left(\frac{\partial}{\partial t}\left(y\left(U_{h}\right)-Y_{h}\right), v\right)+d\left(y\left(U_{h}\right)-Y_{h}, v\right) \\
=L_{1}+L_{2}+L_{3}+\tilde{g}\left(Y_{h}, v\right)-\int_{0}^{t} c\left(t, \tau ; y\left(U_{h}\right)(\tau), v\right) d \tau
\end{gathered}
$$

For $t \in\left[t_{i-1}, t_{i}\right]$, let

$$
\begin{aligned}
& \tilde{g}\left(Y_{h}, v\right)-\int_{0}^{t} c\left(t, \tau ; y\left(U_{h}\right)(\tau), v\right) d \tau \\
& =\sum_{m=1}^{i} \int_{t_{m-1}}^{t_{m}}\left(\left(C\left(t_{i}, t_{m-1}\right)-C(t, \tau)\right) \nabla Y_{h}^{m-1}, \nabla v\right) d \tau+\sum_{m=1}^{i} \int_{t_{m-1}}^{t_{m}} c\left(t, \tau ; Y_{h}^{m-1}-Y_{h}, v\right) d \tau \\
& \quad+\sum_{m=1}^{i} \int_{t_{m-1}}^{t_{m}} c\left(t, \tau ; Y_{h}-y\left(U_{h}\right), v\right) d \tau+\int_{t}^{t_{i}} c\left(t, \tau ; y\left(U_{h}\right)-Y_{h}, v\right) d \tau \\
& \quad+\int_{t}^{t_{i}} c\left(t, \tau ; Y_{h}-\hat{Y}_{h}, v\right) d \tau+\int_{t}^{t_{i}} c\left(t, \tau ; \hat{Y}_{h}, v\right) d \tau
\end{aligned}
$$

Then we set $v=y\left(U_{h}\right)-Y_{h}$ and $v=\frac{\partial}{\partial t}\left(y\left(U_{h}\right)-Y_{h}\right)$ in (4.26) and (4.27), respectively. Similarly to (4.23), integrating in time from 0 to $T$, we obtain: 


$$
\begin{aligned}
&\left\|y\left(U_{h}\right)-Y_{h}\right\|_{L^{\infty}\left(0, T ; L^{2}(\Omega)\right)}^{2}+\left\|y\left(U_{h}\right)-Y_{h}\right\|_{L^{2}\left(0, T ; H^{1}(\Omega)\right)}^{2} \\
& \leq C\left\{\sum _ { i = 1 } ^ { N } k _ { i } \left(\sum _ { \tau } h _ { \tau } ^ { 2 } \int _ { \tau } \left(\hat{f}+U_{h}-\frac{\partial}{\partial t} Y_{h}+\operatorname{div}\left(A \nabla \frac{\partial}{\partial t} Y_{h}\right)+\operatorname{div}\left(D \nabla \hat{Y}_{h}\right)\right.\right.\right. \\
&\left.+\sum_{m=1}^{i} k_{m} \operatorname{div}\left(C\left(t_{i}, t_{m-1}\right) \nabla Y_{h}^{m-1}\right)\right)^{2}+\sum_{l} h_{l} \int_{l}\left[\left(A \nabla \frac{\partial}{\partial t} Y_{h}\right) \cdot n+\left(D \nabla \hat{Y}_{h}\right) \cdot n\right. \\
&\left.\left.+\sum_{m=1}^{i} k_{m}\left(C\left(t_{i}, t_{m-1}\right) \nabla Y_{h}^{m-1}\right) \cdot n\right]^{2}\right)+\|f-\hat{f}\|_{L^{2}\left(0, T ; L^{2}(\Omega)\right)}^{2} \\
&+\sum_{i=1}^{N} \int_{t_{i-1}}^{t_{i}}\left(\sum_{m=1}^{i} \int_{t_{m-1}}^{t_{m}}\left\|\left(C\left(t_{i}, t_{m-1}\right)-C(t, \tau)\right) \nabla Y_{h}^{m-1}\right\|_{0, \Omega}^{2} d \tau\right) d t \\
&+\sum_{i=1}^{N} \frac{k_{i}}{3}\left|Y_{h}^{i-1}-Y_{h}^{i}\right|_{1, \Omega}^{2}+\sum_{i=1}^{N} k_{i} \sum_{m=1}^{i} \frac{k_{m}}{3}\left|Y_{h}^{m-1}-Y_{h}^{m}\right|_{1, \Omega}^{2} \\
&\left.+\sum_{i=1}^{N} k_{i}^{3}\left|\hat{Y}_{h}\right|_{1, \Omega}^{2}+\left\|Y_{h}(x, 0)-y_{0}(x)\right\|_{0, \Omega}^{2}\right\} \\
&= C\left(\eta_{7}^{2}+\cdots+\eta_{13}^{2}\right)
\end{aligned}
$$

and

$$
\left\|\frac{\partial}{\partial t}\left(y\left(U_{h}\right)-Y_{h}\right)\right\|_{L^{2}\left(0, T ; H^{1}(\Omega)\right)}^{2} \leq C\left(\eta_{7}^{2}+\cdots+\eta_{13}^{2}\right) .
$$

Combing (4.23)-(4.24) and (4.28)-(4.29), we can prove Lemma 4.4. This completes the proof.

Lemma 4.5 Suppose that $(y, p, u)$ and $\left(y\left(U_{h}\right), p\left(U_{h}\right)\right)$ are the solutions of (2.4) and (4.3), respectively. Then we have:

$$
\begin{aligned}
\| y & -y\left(U_{h}\right)\left\|_{L^{\infty}\left(0, T ; L^{2}(\Omega)\right)}^{2}+\right\| y-y\left(U_{h}\right) \|_{L^{2}\left(0, T ; H^{1}(\Omega)\right)}^{2} \\
& +\left\|\frac{\partial}{\partial t}\left(y-y\left(U_{h}\right)\right)\right\|_{L^{2}\left(0, T ; H^{1}(\Omega)\right)}^{2} \leq C\left\|u-U_{h}\right\|_{L^{2}\left(0, T ; L^{2}(\Omega)\right)}^{2}, \\
\| p & -p\left(U_{h}\right)\left\|_{L^{\infty}\left(0, T ; L^{2}(\Omega)\right)}^{2}+\right\| p-p\left(U_{h}\right) \|_{L^{2}\left(0, T ; H^{1}(\Omega)\right)}^{2} \\
& +\left\|\frac{\partial}{\partial t}\left(p-p\left(U_{h}\right)\right)\right\|_{L^{2}\left(0, T ; H^{1}(\Omega)\right)}^{2} \leq C\left\|y-y\left(U_{h}\right)\right\|_{L^{2}\left(0, T ; L^{2}(\Omega)\right)}^{2} .
\end{aligned}
$$

Proof Letting $w=y-y\left(U_{h}\right)$ and $w=\frac{\partial}{\partial t}\left(y-y\left(U_{h}\right)\right)$ in (4.8), we have

$$
\begin{aligned}
& \left\|y-y\left(U_{h}\right)\right\|_{L^{\infty}\left(0, T ; L^{2}(\Omega)\right)}^{2}+\left\|y-y\left(U_{h}\right)\right\|_{L^{2}\left(0, T ; H^{1}(\Omega)\right)}^{2} \leq C\left\|u-U_{h}\right\|_{L^{2}\left(0, T ; L^{2}(\Omega)\right)}^{2}, \\
& \left\|\frac{\partial}{\partial t}\left(y-y\left(U_{h}\right)\right)\right\|_{L^{2}\left(0, T ; H^{1}(\Omega)\right)}^{2} \leq C\left\|u-U_{h}\right\|_{L^{2}\left(0, T ; L^{2}(\Omega)\right)}^{2} .
\end{aligned}
$$

We can similarly obtain the estimate for $p-p\left(U_{h}\right)$. This completes the proof. 
We now present an upper bound of the a posteriori error estimates.

Theorem 4.6 Suppose that $(y, p, u)$ and $\left(Y_{h}, P_{h}, U_{h}\right)$ are the solutions of (2.4) and (3.7), respectively. Then we have:

$$
\begin{aligned}
& \left\|u-U_{h}\right\|_{L^{2}\left(0, T ; L^{2}(\Omega)\right)}^{2}+\left\|y-Y_{h}\right\|_{L^{\infty}\left(0, T ; L^{2}(\Omega)\right)}^{2}+\left\|y-Y_{h}\right\|_{L^{2}\left(0, T ; H^{1}(\Omega)\right)}^{2} \\
& +\left\|\frac{\partial}{\partial t}\left(y-Y_{h}\right)\right\|_{L^{2}\left(0, T ; H^{1}(\Omega)\right)}^{2}+\left\|p-P_{h}\right\|_{L^{\infty}\left(0, T ; L^{2}(\Omega)\right)}^{2}+\left\|p-P_{h}\right\|_{L^{2}\left(0, T ; H^{1}(\Omega)\right)}^{2} \\
& +\left\|\frac{\partial}{\partial t}\left(p-P_{h}\right)\right\|_{L^{2}\left(0, T ; H^{1}(\Omega)\right)} \leq C \sum_{i=1}^{13} \eta_{i}^{2},
\end{aligned}
$$

where $\eta_{i}(i=1, \ldots, 13)$ is defined in (4.5) and (4.12)

Proof From Lemmas 4.3 and 4.4 we have

$$
\left\|u-U_{h}\right\|_{L^{2}\left(0, T ; L^{2}(\Omega)\right)}^{2} \leq C \eta_{1}^{2}+C\left\|P_{h}-p\left(U_{h}\right)\right\|_{L^{2}\left(0, T ; L^{2}(\Omega)\right)}^{2} \leq C \sum_{i=1}^{13} \eta_{i}^{2} .
$$

By the triangle inequality we have

$$
\begin{aligned}
\| y- & Y_{h}\left\|_{L^{\infty}\left(0, T ; L^{2}(\Omega)\right)}^{2}+\right\| y-Y_{h}\left\|_{L^{2}\left(0, T ; H^{1}(\Omega)\right)}^{2}+\right\| \frac{\partial}{\partial t}\left(y-Y_{h}\right) \|_{L^{2}\left(0, T ; H^{1}(\Omega)\right)}^{2} \\
\leq & \left\|y-y\left(U_{h}\right)\right\|_{L^{\infty}\left(0, T ; L^{2}(\Omega)\right)}^{2}+\left\|y-y\left(U_{h}\right)\right\|_{L^{2}\left(0, T ; H^{1}(\Omega)\right)}^{2} \\
& +\left\|\frac{\partial}{\partial t}\left(y-y\left(U_{h}\right)\right)\right\|_{L^{2}\left(0, T ; H^{1}(\Omega)\right)}^{2}+\left\|y\left(U_{h}\right)-Y_{h}\right\|_{L^{\infty}\left(0, T ; L^{2}(\Omega)\right)}^{2} \\
& +\left\|y\left(U_{h}\right)-Y_{h}\right\|_{L^{2}\left(0, T ; H^{1}(\Omega)\right)}^{2}+\left\|\frac{\partial}{\partial t}\left(y\left(U_{h}\right)-Y_{h}\right)\right\|_{L^{2}\left(0, T ; H^{-1}(\Omega)\right)}^{2} .
\end{aligned}
$$

We have the same results for $p-P_{h}$. Then from (4.35), Lemmas 4.3-4.5, combined with (4.34), we obtain (4.33). This completes the proof.

\subsection{Lower bound estimates}

For convenience, we first define the average integration of $v$ in element $\tau,\left.\bar{v}\right|_{\tau}=\frac{\int_{\tau} v}{\int_{\tau} 1}$, and define $R_{\tau}, R_{l}, T_{\tau}, T_{l}$ as follows:

$$
\begin{aligned}
& R_{\tau}=\hat{f}+U_{h}-\frac{\partial}{\partial t} Y_{h}+\operatorname{div}\left(A \nabla \frac{\partial}{\partial t} Y_{h}\right)+\operatorname{div}\left(D \nabla \hat{Y}_{h}\right)+\sum_{m=1}^{i} k_{m} \operatorname{div}\left(C\left(t_{i}, t_{m-1}\right) \nabla Y_{h}^{m-1}\right), \\
& R_{l}=\left[\left(A \nabla \frac{\partial}{\partial t} Y_{h}\right) \cdot n+\left(D \nabla \hat{Y}_{h}\right) \cdot n+\sum_{m=1}^{i} k_{m}\left(C\left(t_{i}, t_{m-1}\right) \nabla Y_{h}^{m-1}\right) \cdot n\right], \\
& T_{\tau}=\hat{Y}_{h}-z_{d}+\frac{\partial}{\partial t} P_{h}-\operatorname{div}\left(A^{*} \nabla \frac{\partial}{\partial t} P_{h}\right)+\operatorname{div}\left(D^{*} \nabla \tilde{P}_{h}\right)+\sum_{m=i}^{N} k_{m} \operatorname{div}\left(C^{*}\left(t_{m}, t_{i-1}\right) \nabla P_{h}^{m}\right), \\
& T_{l}=\left[\left(A^{*} \nabla \frac{\partial}{\partial t} P_{h}\right) \cdot n-\left(D^{*} \nabla \tilde{P}_{h}\right) \cdot n-\sum_{m=i}^{N} k_{m}\left(C^{*}\left(t_{m}, t_{i-1}\right) \nabla P_{h}^{m}\right) \cdot n\right] .
\end{aligned}
$$


In order to present the main conclusion in this subsection, we first use the standard bubble function technique in $[11,26]$ and give the following lemma.

Lemma 4.7 Suppose that $(y, p, u)$ and $\left(Y_{h}, P_{h}, U_{h}\right)$ are the solutions of $(2.4)$ and (3.7), respectively. Then we have:

$$
\begin{aligned}
\eta_{7}^{2}+\eta_{9}^{2}+\eta_{11}^{2} \leq & C\left\{\|f-\hat{f}\|_{L^{2}\left(0, T ; H^{-1}(\Omega)\right)}^{2}+\left\|u-U_{h}\right\|_{L^{2}\left(0, T ; L^{2}(\Omega)\right)}^{2}\right. \\
& +\left\|y-Y_{h}\right\|_{L^{2}\left(0, T ; H^{1}(\Omega)\right)}^{2}+\left\|\frac{\partial}{\partial t}\left(y-Y_{h}\right)\right\|_{L^{2}\left(0, T ; H^{1}(\Omega)\right)}^{2}+\sum_{i=1}^{N} k_{i}^{3}\left|\hat{Y}_{h}\right|_{1, \Omega}^{2} \\
& +\sum_{i=1}^{N} \int_{t_{i-1}}^{t_{i}}\left(\sum_{m=1}^{i} \int_{t_{m-1}}^{t_{m}}\left\|\left(C\left(t_{i}, t_{m-1}\right)-C(t, \tau)\right) \nabla Y_{h}^{m-1}\right\|_{0, \Omega}^{2} d \tau\right) d t \\
& \left.+\sum_{i=1}^{N} k_{i} \sum_{\tau \in T^{h}} \int_{\tau} h_{\tau}^{2}\left(R_{\tau}-\bar{R}_{\tau}\right)^{2}+\sum_{i=1}^{N} k_{i} \sum_{l \in \tilde{\varepsilon}^{h}} \int_{l} h_{l}\left(R_{l}-\bar{R}_{l}\right)^{2}\right\} .
\end{aligned}
$$

Proof By using techniques in [23] we prove the lemma in three steps.

(1) First, we estimate $\frac{k_{i}}{3}\left|Y_{h}^{i-1}-Y_{h}^{i}\right|_{1, \Omega}^{2}$.

Let

$$
\eta_{2 i}^{2}=\sum_{\tau} h_{\tau}^{2} \int_{\tau} R_{\tau}^{2}+\sum_{l} h_{l} \int_{l} R_{l}^{2}
$$

By using the equalities $\frac{k_{i}}{3}\left|Y_{h}^{i-1}-Y_{h}^{i}\right|_{1, \Omega}^{2}=\int_{t_{i-1}}^{t_{i}}\left|Y_{h}-\hat{Y}_{h}\right|_{1, \Omega}^{2} d t$ and

$$
\begin{aligned}
\left(\frac{\partial}{\partial t}\left(y-Y_{h}\right), v\right)+a\left(\frac{\partial}{\partial t}\left(y-Y_{h}\right), v\right)+d\left(y-Y_{h}, v\right)+\int_{0}^{t} c(t, \tau ; y(\tau), v) d \tau-\tilde{g}\left(Y_{h}, v\right) \\
=\left(\frac{\partial}{\partial t}\left(y-Y_{h}\right), v\right)+a\left(\frac{\partial}{\partial t}\left(y-Y_{h}\right), v\right)+d\left(y-Y_{h}, v\right)+\int_{0}^{t} c(t, \tau ; y(\tau), v) d \tau \\
\quad-\sum_{m=1}^{i} k_{m} c\left(t_{i}, t_{m-1} ; Y_{h}^{m-1}, v\right) \\
=\left(f+u-\hat{f}-U_{h}, v\right)+\left(\hat{f}+U_{h}, v-\pi_{h} v\right)-\left(\frac{\partial}{\partial t} Y_{h}, v-\pi_{h} v\right)-a\left(\frac{\partial}{\partial t} Y_{h}, v-\pi_{h} v\right) \\
\quad-d\left(\hat{Y}_{h}, v-\pi_{h} v\right)-\sum_{m=1}^{i} k_{m} c\left(t_{i}, t_{m-1} ; Y_{h}^{m-1}, v-\pi_{h} v\right)-d\left(Y_{h}-\hat{Y}_{h}, v\right)
\end{aligned}
$$

we have

$$
\begin{aligned}
d\left(Y_{h}-\hat{Y}_{h}, v\right) & \\
= & (f-\hat{f}, v)+\left(u-U_{h}, v\right)+\left\{\sum _ { \tau } \int _ { \tau } \left(\hat{f}+U_{h}-\frac{\partial}{\partial t} Y_{h}+\operatorname{div}\left(A \nabla \frac{\partial}{\partial t} Y_{h}\right)+\operatorname{div}\left(D \nabla \hat{Y}_{h}\right)\right.\right. \\
& \left.+\sum_{m=1}^{i} k_{m} \operatorname{div}\left(C\left(t_{i}, t_{m-1}\right)\right) \nabla Y_{h}^{m-1}\right)-\sum_{l} \int_{l}\left[\left(A \nabla \frac{\partial}{\partial t} Y_{h}\right) \cdot n+\left(D \nabla \hat{Y}_{h}\right) \cdot n\right.
\end{aligned}
$$




$$
\begin{aligned}
& \left.\left.\left.+\sum_{m=1}^{i} k_{m}\left(C\left(t_{i}, t_{m-1}\right)\right) \nabla Y_{h}^{m-1}\right) \cdot n\right]\right\}\left(v-\Pi_{h} v\right) \\
& -\left(\frac{\partial}{\partial t}\left(y-Y_{h}\right), v\right)-a\left(\frac{\partial}{\partial t}\left(y-Y_{h}\right), v\right) \\
& -d\left(y-Y_{h}, v\right)+\sum_{m=1}^{i} k_{m} c\left(t_{i}, t_{m-1} ; Y_{h}^{m-1}, v\right)-\int_{0}^{t} c(t, \tau ; y(\tau), v) d \tau
\end{aligned}
$$

Then

$$
\begin{aligned}
\int_{t_{i-1}}^{t_{i}} d & \left(Y_{h}-\hat{Y}_{h}, v\right) d t \\
\leq & C\left\{\|f-\hat{f}\|_{L^{2}\left(t_{i-1}, t_{i} ; H^{-1}(\Omega)\right)}^{2}+\left\|u-U_{h}\right\|_{L^{2}\left(t_{i-1}, t_{i} ; L^{2}(\Omega)\right)}^{2}\right. \\
& +k_{i}\left\{\sum _ { \tau \in T ^ { h } } \int _ { \tau } h _ { \tau } ^ { 2 } \left(\hat{f}+U_{h}-\frac{\partial}{\partial t} Y_{h}+\operatorname{div}\left(A \nabla \frac{\partial}{\partial t} Y_{h}\right)+\operatorname{div}\left(D \nabla \hat{Y}_{h}\right)+\operatorname{div}\left(A \nabla \hat{Y}_{h}\right)\right.\right. \\
& \left.+\sum_{m=1}^{i} k_{m} \operatorname{div}\left(C\left(t_{i}, t_{m-1}\right) \nabla Y_{h}^{m-1}\right)\right)^{2}+\sum_{l} \int_{l} h_{l}\left[\left(A \nabla \frac{\partial}{\partial t} Y_{h}\right) \cdot n+\left(D \nabla \hat{Y}_{h}\right) \cdot n\right. \\
& \left.\left.\left.+\sum_{m=1}^{i} k_{m}\left(C\left(t_{i}, t_{m-1}\right) \nabla Y_{h}^{m-1}\right) \cdot n\right]^{2}\right\}+\left\|y-Y_{h}\right\|_{L^{2}\left(t_{i-1}, t_{i} ; H^{1}(\Omega)\right)}^{2}\right) \\
& \left.+\left\|\frac{\partial}{\partial t}\left(y-Y_{h}\right)\right\|_{L^{2}\left(t_{i-1}, t_{i} ; H^{1}(\Omega)\right)}^{2}\right\}+\varepsilon|v|_{L^{2}\left(t_{i-1}, t_{i} ; H^{1}(\Omega)\right)}^{t} \\
& +\int_{t_{i-1}}^{t_{i}}\left\{\sum_{m=1}^{i} k_{m} c\left(t_{i}, t_{m-1} ; Y_{h}^{m-1}, v\right)-\int_{0}^{c} c(t, \tau ; y(\tau), v) d \tau\right\} d t .
\end{aligned}
$$

Letting

$$
\begin{aligned}
J= & \int_{t_{i-1}}^{t_{i}}\left\{\sum_{m=1}^{i} k_{m} c\left(t_{i}, t_{m-1} ; Y_{h}^{m-1}, v\right)-\int_{0}^{t} c(t, \tau ; y(\tau), v) d \tau\right\} d t \\
= & \int_{t_{i-1}}^{t_{i}}\left\{\sum_{m=1}^{i} \int_{t_{m-1}}^{t_{m}}\left(\left(C\left(t_{i}, t_{m-1}\right)-C(t, \tau)\right) \nabla Y_{h}^{m-1}, \nabla v\right) d \tau\right\} d t \\
& +\int_{t_{i-1}}^{t_{i}}\left\{\sum_{m=1}^{i} \int_{t_{m-1}}^{t_{m}} c\left(t, \tau ; Y_{h}^{m-1}-Y_{h}, v\right) d \tau\right\} d t \\
& +\int_{t_{i-1}}^{t_{i}}\left\{\sum_{m=1}^{i} \int_{t_{m-1}}^{t_{m}} c\left(t, \tau ;\left(Y_{h}-y\right)(\tau), v\right) d \tau\right\} d t \\
& +\int_{t_{i-1}}^{t_{i}} \int_{t}^{t_{i}} c\left(t, \tau ;\left(y-Y_{h}\right)(\tau), v\right) d \tau d t+\int_{t_{i-1}}^{t_{i}} \int_{t}^{t_{i}} c\left(t, \tau ;\left(Y_{h}-\hat{Y}_{h}\right)(\tau), v\right) d \tau d t \\
& +\int_{t_{i-1}}^{t_{i}} \int_{t}^{t_{i}} c\left(t, \tau ; \hat{Y}_{h}, v\right) d \tau d t \\
= & J_{1}+\cdots+J_{6},
\end{aligned}
$$


we have

$$
\begin{aligned}
J_{1} \leq & C \int_{t_{i-1}}^{t_{i}}\left\{\sum_{m=1}^{i} \int_{t_{m-1}}^{t_{m}}\left\|\left(C\left(t_{i}, t_{m-1}\right)-C(t, \tau)\right) \nabla Y_{h}^{m-1}\right\|_{0, \Omega}^{2} d \tau\right\} d t \\
& +\varepsilon|v|_{L^{2}\left(t_{i-1}, t_{i} ; H^{1}(\Omega)\right)^{\prime}}^{2} \\
J_{2} \leq & C k_{i} \sum_{m=1}^{i} \frac{k_{m}}{3}\left|Y_{h}^{m-1}-Y_{h}^{m}\right|_{1, \Omega}^{2}+\varepsilon|v|_{L^{2}\left(t_{i-1}, t_{i} ; H^{1}(\Omega)\right)}^{2}, \\
J_{3}= & \int_{t_{i-1}}^{t_{i}}\left\{\int_{0}^{t_{i}} c\left(t, \tau ;\left(Y_{h}-y\right)(\tau), v\right) d \tau\right\} d t \\
= & \int_{0}^{t_{i}}\left(\int_{t_{i-1}}^{t_{i}} c\left(\tau, t ;\left(Y_{h}-y\right)(t), v(\tau)\right) d \tau\right) d t \\
& \leq C k_{i}\left\|Y_{h}-y\right\|_{L^{2}\left(0, t_{i} ; H^{1}(\Omega)\right)}^{2}+\varepsilon|v|_{L^{2}\left(t_{i-1}, t_{i} ; H^{1}(\Omega)\right)^{\prime}}^{2} \\
J_{4} \leq & C k_{i}\left\|y-Y_{h}\right\|_{L^{2}\left(t_{i-1}, t_{i} ; H^{1}(\Omega)\right)}^{2}+\varepsilon|v|_{L^{2}\left(t_{i-1}, t_{i} ; H^{1}(\Omega)\right)^{\prime}}^{2} \\
J_{5} \leq & C \frac{k_{i}^{2}}{3}\left|Y_{h}^{i-1}-Y_{h}^{i}\right|_{1, \Omega}^{2}+\varepsilon|v|_{L^{2}\left(t_{i-1}, t_{i} ; H^{1}(\Omega)\right)}^{2} \\
J_{6} \leq & C k_{i}^{3}\left|\hat{Y}_{h}\right|_{1, \Omega}^{2}+\varepsilon|v|_{L^{2}\left(t_{i-1}, t_{i} ; H^{1}(\Omega)\right)}^{2} .
\end{aligned}
$$

Setting $v=Y_{h}-\hat{Y}_{h}$ in (4.39), from (4.38)-(4.42) we obtain:

$$
\begin{aligned}
\frac{k_{i}}{3}\left|Y_{h}^{i-1}-Y_{h}^{i}\right|_{1, \Omega}^{2}= & \int_{t_{i-1}}^{t_{i}}\left|Y_{h}-\hat{Y}_{h}\right|_{1, \Omega}^{2} d t \\
\leq & C \int_{t_{i-1}}^{t_{i}} d\left(Y_{h}-\hat{Y}_{h}, Y_{h}-\hat{Y}_{h}\right) d t \\
\leq & C\left\{\|f-\hat{f}\|_{L^{2}\left(t_{i-1}, t_{i} ; H^{-1}(\Omega)\right)}^{2}+\left\|u-U_{h}\right\|_{L^{2}\left(t_{i-1}, t_{i} L^{2}(\Omega)\right)}^{2}+k_{i} \eta_{2 i}^{2}\right. \\
& +\left\|y-Y_{h}\right\|_{L^{2}\left(t_{i-1}, t_{i} ; H^{1}(\Omega)\right)}^{2}+\left\|\frac{\partial}{\partial t}\left(y-Y_{h}\right)\right\|_{L^{2}\left(t_{i-1}, t_{i} ; H^{1}(\Omega)\right)}^{2} \\
& +\int_{t_{i-1}}^{t_{i}}\left\{\sum_{m=1}^{i} \int_{t_{m-1}}^{t_{m}}\left\|\left(C\left(t_{i}, t_{m-1}\right)-C(t, \tau)\right) \nabla Y_{h}^{m-1}\right\|_{0, \Omega}^{2} d \tau\right\} d t \\
& +k_{i} \sum_{m=1}^{i} \frac{k_{m}}{3}\left|Y_{h}^{m-1}-Y_{h}^{m}\right|_{1, \Omega}^{2} \\
& \left.+k_{i}^{3}\left|\hat{Y}_{h}\right|_{1, \Omega}^{2}+k_{i}\left\|y-Y_{h}\right\|_{L^{2}\left(0, t_{i} ; H^{1}(\Omega)\right)}\right\} .
\end{aligned}
$$

(2) Using the bubble function methods in [11, 26] and the techniques in [23], we have:

$$
\begin{aligned}
k_{i} \eta_{2 i}^{2} \leq & C\left\{\|f-\hat{f}\|_{L^{2}\left(t_{i-1}, t_{i} ; H^{-1}(\Omega)\right)}^{2}+\left\|u-U_{h}\right\|_{L^{2}\left(t_{i-1}, t_{i} L^{2}(\Omega)\right)}^{2}\right. \\
& +\left\|y-Y_{h}\right\|_{L^{2}\left(t_{i-1}, t_{i} ; H^{1}(\Omega)\right)}^{2}+\left\|\frac{\partial}{\partial t}\left(y-Y_{h}\right)\right\|_{L^{2}\left(t_{i-1}, t_{i} ; H^{1}(\Omega)\right)}^{2}
\end{aligned}
$$




$$
\begin{aligned}
& +\int_{t_{i-1}}^{t_{i}}\left(\sum_{m=1}^{i} \int_{t_{m-1}}^{t_{m}}\left\|\left(C\left(t_{i}, t_{m-1}\right)-C(t, \tau)\right) \nabla Y_{h}^{m-1}\right\|_{0, \Omega}^{2} d \tau\right) d t \\
& \left.+k_{i} \sum_{m=1}^{i} \frac{k_{m}}{3}\left|Y_{h}^{m-1}-Y_{h}^{m}\right|_{1, \Omega}^{2}+k_{i}^{3}\left|\hat{Y}_{h}\right|_{1, \Omega}^{2}+k_{i}\left\|y-Y_{h}\right\|_{L^{2}\left(0, t_{i} ; H^{1}(\Omega)\right)}^{2}\right\} \\
& +C_{\delta} k_{i}\left(\sum_{\tau \in T^{h}} \int_{\tau} h_{\tau}^{2}\left(R_{\tau}-\bar{R}_{\tau}\right)^{2}+\sum_{l \in \tilde{\varepsilon}^{h}} \int_{l} h_{l}\left(R_{l}-\bar{R}_{l}\right)^{2}\right) .
\end{aligned}
$$

(3) From (4.43) and (4.44) we obtain:

$$
\begin{aligned}
& \frac{k_{i}}{3}\left|Y_{h}^{i-1}-Y_{h}^{i}\right|_{1, \Omega}^{2} \\
& \leq C\left\{\|f-\hat{f}\|_{L^{2}\left(t_{i-1}, t_{i} ; H^{-1}(\Omega)\right)}^{2}+\left\|u-U_{h}\right\|_{L^{2}\left(t_{i-1}, t_{i} ; L^{2}(\Omega)\right)}^{2}\right. \\
& \quad+\left\|y-Y_{h}\right\|_{L^{2}\left(t_{i-1}, t_{i} ; H^{1}(\Omega)\right)}^{2}+\left\|\frac{\partial}{\partial t}\left(y-Y_{h}\right)\right\|_{L^{2}\left(t_{i-1}, t_{i} ; H^{1}(\Omega)\right)}^{2} \\
& \quad+\int_{t_{i-1}}^{t_{i}}\left(\sum_{m=1}^{i} \int_{t_{m-1}}^{t_{m}}\left\|\left(C\left(t_{i}, t_{m-1}\right)-C(t, \tau)\right) \nabla Y_{h}^{m-1}\right\|_{0, \Omega}^{2} d \tau\right) d t \\
& \quad+k_{i} \sum_{m=1}^{i} \frac{k_{m}}{3}\left|Y_{h}^{m-1}-Y_{h}^{m}\right|_{1, \Omega}^{2}+k_{i}^{3}\left|\hat{Y}_{h}\right|_{1, \Omega}^{2}+k_{i}\left\|y-Y_{h}\right\|_{L^{2}\left(0, t_{i} ; H^{1}(\Omega)\right)}^{2} \\
& \left.\quad+k_{i}\left(\sum_{\tau \in T^{h}} \int_{\tau} h_{\tau}^{2}\left(R_{\tau}-\bar{R}_{\tau}\right)^{2}+\sum_{l \in \tilde{\varepsilon}_{h}} \int_{l} h_{l}\left(R_{l}-\bar{R}_{l}\right)^{2}\right)\right\}
\end{aligned}
$$

Further, from the Gronwall inequality, combined with (4.44), we have

$$
\begin{aligned}
\eta_{7}^{2}+ & \eta_{9}^{2}+\eta_{11}^{2} \\
\leq & \sum_{i=1}^{N} k_{i} \eta_{2 i}^{2}+(1+T) \sum_{i=1}^{N} \frac{k_{i}}{3}\left|Y_{h}^{i-1}-Y_{h}^{i}\right|_{1, \Omega}^{2} \\
\leq & C\left\{\|f-\hat{f}\|_{L^{2}\left(0, T ; H^{-1}(\Omega)\right)}^{2}+\left\|u-U_{h}\right\|_{L^{2}\left(0, T ; L^{2}(\Omega)\right)}^{2}+\left\|y-Y_{h}\right\|_{L^{2}\left(0, T ; H^{1}(\Omega)\right)}^{2}\right. \\
& +\left\|\frac{\partial}{\partial t}\left(y-Y_{h}\right)\right\|_{L^{2}\left(0, T ; H^{1}(\Omega)\right)}^{2}+\sum_{i=1}^{N} k_{i}^{3}\left|\hat{Y}_{h}\right|_{1, \Omega}^{2} \\
& +\sum_{i=1}^{N} \int_{t_{i-1}}^{t_{i}}\left(\sum_{m=1}^{i} \int_{t_{m-1}}^{t_{m}}\left\|\left(C\left(t_{i}, t_{m-1}\right)-C(t, \tau)\right) \nabla Y_{h}^{m-1}\right\|_{0, \Omega}^{2} d \tau\right) d t \\
& \left.+\sum_{i=1}^{N} k_{i}\left(\sum_{\tau \in T^{h}} \int_{\tau} h_{\tau}^{2}\left(R_{\tau}-\bar{R}_{\tau}\right)^{2}+\sum_{l \in \tilde{\varepsilon}^{h}} \int_{l} h_{l}\left(R_{l}-\bar{R}_{l}\right)^{2}\right)\right\} .
\end{aligned}
$$

This completes the proof.

Similarly, we can also obtain the following lemma. 
Lemma 4.8 Suppose that $(y, p, u)$ and $\left(Y_{h}, P_{h}, U_{h}\right)$ are the solutions of (2.4) and (3.7), respectively. Then we have:

$$
\begin{aligned}
\eta_{2}^{2}+\eta_{3}^{2}+\eta_{5}^{2} \leq & C\left\{\left\|y-Y_{h}\right\|_{L^{2}\left(0, T ; H^{1}(\Omega)\right)}^{2}+\left\|p-P_{h}\right\|_{L^{2}\left(0, T ; H^{1}(\Omega)\right)}^{2}\right. \\
& +\left\|\frac{\partial}{\partial t}\left(p-P_{h}\right)\right\|_{L^{2}\left(0, T ; H^{1}(\Omega)\right)}^{2}+\sum_{i=1}^{N} k_{i}^{3}\left|\tilde{P}_{h}\right|_{1, \Omega}^{2} \\
& +\sum_{i=1}^{N} \int_{t_{i-1}}^{t_{i}}\left(\sum_{m=i}^{N} \int_{t_{m-1}}^{t_{m}}\left\|\left(C^{*}\left(t_{m}, t_{i-1}\right)-C^{*}(t, \tau)\right) \nabla P_{h}^{m}\right\|_{0, \Omega}^{2} d \tau\right) d t \\
& \left.+\sum_{i=1}^{N} k_{i} \sum_{\tau \in T^{h}} \int_{\tau} h_{\tau}^{2}\left(T_{\tau}-\bar{T}_{\tau}\right)^{2}+\sum_{i=1}^{N} k_{i} \sum_{l \in \tilde{\varepsilon}^{h}} \int_{l} h_{l}\left(T_{l}-\bar{T}_{l}\right)^{2}\right\} .
\end{aligned}
$$

Lemma 4.9 Suppose that $(y, p, u)$ and $\left(Y_{h}, P_{h}, U_{h}\right)$ are the solutions of (2.4) and (3.7), respectively. Then we have:

$$
\eta_{1}^{2} \leq C\left\{\left\|u-U_{h}\right\|_{L^{2}\left(0, T ; L^{2}(\Omega)\right)}^{2}+\left\|p-P_{h}\right\|_{L^{2}\left(0, T ; L^{2}(\Omega)\right)}^{2}\right\}
$$

Therefore, from Lemmas 4.3-4.8 we can easily obtain the following theorem.

Theorem 4.10 Suppose that $(y, p, u)$ and $\left(Y_{h}, P_{h}, U_{h}\right)$ are the solutions of (2.4) and (3.7), respectively. Then we have the following estimates:

$$
\begin{aligned}
\eta_{1}^{2}+ & \eta_{2}^{2}+\eta_{3}^{2}+\eta_{5}^{2}+\eta_{7}^{2}+\eta_{9}^{2}+\eta_{11}^{2} \\
\leq & C\left\{\left\|u-U_{h}\right\|_{L^{2}\left(0, T ; L^{2}(\Omega)\right)}^{2}+\left\|y-Y_{h}\right\|_{L^{2}\left(0, T ; H^{1}(\Omega)\right)}^{2}+\left\|y-Y_{h}\right\|_{L^{\infty}\left(0, T ; L^{2}(\Omega)\right)}^{2}\right. \\
& +\left\|\frac{\partial}{\partial t}\left(y-Y_{h}\right)\right\|_{L^{2}\left(0, T ; H^{1}(\Omega)\right)}^{2}+\left\|p-P_{h}\right\|_{L^{2}\left(0, T ; H^{1}(\Omega)\right)}^{2}+\left\|p-P_{h}\right\|_{L^{\infty}\left(0, T ; L^{2}(\Omega)\right)}^{2} \\
& +\left\|\frac{\partial}{\partial t}\left(p-P_{h}\right)\right\|_{L^{2}\left(0, T ; H^{1}(\Omega)\right)}^{2}+\|f-\hat{f}\|_{L^{2}\left(0, T ; H^{-1}(\Omega)\right)}^{2} \\
& +\sum_{i=1}^{N} \int_{t_{i-1}}^{t_{i}}\left(\sum_{m=1}^{i} \int_{t_{m-1}}^{t_{m}}\left\|\left(C\left(t_{i}, t_{m-1}\right)-C(t, \tau)\right) \nabla Y_{h}^{m-1}\right\|_{0, \Omega}^{2} d \tau\right) d t \\
& +\sum_{i=1}^{N} \int_{t_{i-1}}^{t_{i}}\left(\sum_{m=i}^{N} \int_{t_{m-1}}^{t_{m}}\left\|\left(C^{*}\left(t_{m}, t_{i-1}\right)-C^{*}(t, \tau)\right) \nabla P_{h}^{m}\right\|_{0, \Omega}^{2} d \tau\right) d t \\
& +\sum_{i=1}^{N} k_{i}^{3}\left|\hat{Y}_{h}\right|_{1, \Omega}^{2}+\sum_{i=1}^{N} k_{i}^{3}\left|\tilde{P}_{h}\right|_{1, \Omega}^{2}+\sum_{i=1}^{N} k_{i} \sum_{\tau \in T^{h}} \int_{\tau} h_{\tau}^{2}\left(R_{\tau}-\bar{R}_{\tau}\right)^{2} \\
& +\sum_{i=1}^{N} k_{i} \sum_{\tau \in T^{h}} \int_{\tau} h_{\tau}^{2}\left(T_{\tau}-\bar{T}_{\tau}\right)^{2} \\
& \left.+\sum_{i=1}^{N} k_{i} \sum_{l \in \tilde{\varepsilon}^{h}} \int_{l} h_{l}\left(R_{l}-\bar{R}_{l}\right)^{2}+\sum_{i=1}^{N} k_{i} \sum_{l \in \tilde{\varepsilon}^{h}} \int_{l} h_{l}\left(T_{l}-\bar{T}_{l}\right)^{2}\right\} .
\end{aligned}
$$




\section{Numerical experiment}

In this section, we carry out a numerical experiment to verify the a posteriori error estimates derived in Section 4 . Let $\Omega=\Omega_{U}=(0,1)^{2}$. In order to approximate the state and costate, we use linear FE spaces, and similarly to approximate the control, we use the piecewise constant finite element spaces. We also choose the Euler backward-difference procedure to compute the full-discrete system for time variable.

Here we numerically compute the following quasi-parabolic IDPDE:

$$
\begin{aligned}
& \min _{\int_{\Omega} u \geq 0} \frac{1}{2} \int_{0}^{1}\left\{\int_{\Omega}\left(y-z_{d}\right)^{2}+\int_{\Omega}\left(u-u_{0}\right)^{2}\right\} d t, \\
& \left\{\begin{array}{l}
y_{t}-\Delta y-\Delta y_{t}-\int_{0}^{t}(t-\tau) \Delta y d \tau=f+u, \quad x \in \Omega, 0<t \leq 1, \\
\left.y\right|_{\partial \Omega}=0
\end{array}\right.
\end{aligned}
$$

The exact solution of (5.1)-(5.2) is as follows:

$$
\left\{\begin{array}{l}
p=-(T-t) \sin \pi x_{1} \sin \pi x_{2}, \quad T=1, \\
u_{0}=\left\{\begin{array}{l}
-p, \quad x_{1}+x_{2} \leq 1.0, \\
0.0, \quad x_{1}+x_{2}>1.0,
\end{array}\right. \\
u=u_{0}-p+\max \left(\overline{p-u_{0}}, 0\right), \\
y=t x_{1}\left(1-x_{1}\right) x_{2}\left(1-x_{2}\right), \\
z_{d}=y+p_{t}+\Delta p+\Delta p_{t}+\int_{t}^{T}(t-\tau) \Delta p d \tau, \\
f=y_{t}-\Delta y-\Delta y_{t}-\int_{0}^{t}(t-\tau) \Delta y d \tau-u .
\end{array}\right.
$$

Here we use the AFEpack software package introduced in [27] to conduct our numerical experiment. The experiment is conducted on a uniform mesh and an adaptive mesh. We use the same time step $d t=0.05$ for $u, p, y$. The initial mesh for adaptive mesh is shown in Figure 1 , and the tolerance for the adaptive mesh is $10^{-6}$. We have used the estimators $\eta_{1}$, $\eta_{2}, \eta_{3}, \eta_{5}, \eta_{7}, \eta_{9}, \eta_{11}$, whereas the others are higher-order terms, so omitted traditionally. We use $\eta_{1}$ for adaptive control and use $\eta_{2}, \eta_{3}, \eta_{5}, \eta_{7}, \eta_{9}, \eta_{11}$ for state and costate adaptive, respectively.

In Table 1, we present two different approximation error results for control $u$, state $y$, and costate $p$, where $E_{u}=u-u_{h}, E_{y}=y-y_{h}, E_{p}=p-p_{h}, L^{2}\left(L^{2}\right)$ is $L^{2}\left(0, T ; L^{2}(\Omega)\right), L^{2}\left(H^{1}\right)$

Figure 1 The initial mesh for $u, y$, and $p$.

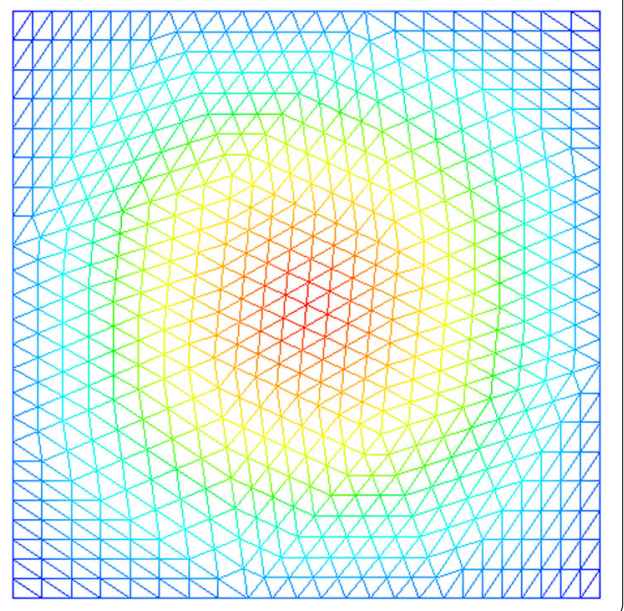


Table 1 Numerical result with time step $d t=0.05$

\begin{tabular}{|c|c|c|c|c|c|c|}
\hline \multicolumn{4}{|c|}{ On uniform mesh } & \multicolumn{3}{|c|}{ On adaptive mesh } \\
\hline N & 50,421 & 50,421 & 50,421 & 22,769 & 3,549 & 3,549 \\
\hline S & 147,168 & 147,168 & 147,168 & 64,463 & 9,576 & 9,576 \\
\hline E & 96,768 & 96,768 & 96,768 & 41,715 & 6,048 & 6,048 \\
\hline error & $\begin{array}{l}\left\|E_{u}\right\|_{L^{2}\left(L^{2}\right)} \\
8.33 \mathrm{e}-02\end{array}$ & $\begin{array}{l}\left\|E_{y}\right\|_{L^{2}\left(H^{1}\right)} \\
1.28 \mathrm{e}-02\end{array}$ & $\begin{array}{l}\left\|E_{p}\right\|_{L^{2}\left(H^{1}\right)} \\
4.75 \mathrm{e}-01\end{array}$ & $\begin{array}{l}\left\|E_{u}\right\|_{L^{2}\left(L^{2}\right)} \\
8.16 \mathrm{e}-02\end{array}$ & $\begin{array}{l}\left\|E_{y}\right\|_{L^{2}\left(H^{1}\right)} \\
5.06 \mathrm{e}-02\end{array}$ & $\begin{array}{l}\left\|E_{p}\right\|_{L^{2}\left(H^{1}\right)} \\
770 \mathrm{e}-01\end{array}$ \\
\hline error & $\begin{array}{l}\left\|E_{u}\right\|_{L^{2}\left(L^{2}\right)} \\
8.33 \mathrm{e}-02\end{array}$ & $\begin{array}{l}\left\|E_{y}\right\|_{L^{2}\left(L^{2}\right)} \\
2.79 e-04\end{array}$ & $\begin{array}{l}\left\|E_{p}\right\|_{L^{2}\left(L^{2}\right)} \\
1.01 \mathrm{e}-01\end{array}$ & $\begin{array}{l}\left\|E_{u}\right\|_{L^{2}\left(L^{2}\right)} \\
8.16 \mathrm{e}-02\end{array}$ & $\begin{array}{l}\left\|E_{y}\right\|_{L^{2}\left(L^{2}\right)} \\
2.09 \mathrm{e}-03\end{array}$ & $\begin{array}{l}\left\|E_{p}\right\|_{L^{2}\left(L^{2}\right)} \\
1.14 \mathrm{e}-01\end{array}$ \\
\hline error & $\begin{array}{l}\left\|E_{u}\right\|_{L \infty}{ }_{\left(L^{2}\right)} \\
3.54 \mathrm{e}-02\end{array}$ & $\begin{array}{l}\left\|E_{y}\right\|_{\left.L_{(\infty}{ }^{2}\right)} \\
3.88 \mathrm{e}-04\end{array}$ & $\begin{array}{l}\left\|E_{p}\right\|_{L \infty\left(L^{2}\right)} \\
2.53 \mathrm{e}-02\end{array}$ & $\begin{array}{l}\left\|E_{U}\right\|_{L \infty}{ }_{\left(L^{2}\right)} \\
3.53 \mathrm{e}-02\end{array}$ & $\begin{array}{l}\left\|E_{y}\right\|_{L \infty\left(L^{2}\right)} \\
5.85 \mathrm{e}-04\end{array}$ & $\begin{array}{l}\left\|E_{p}\right\|_{L \infty\left(L^{2}\right)} \\
3.21 \mathrm{e}-02\end{array}$ \\
\hline
\end{tabular}

Figure 2 Adaptive mesh for the control and the approximation value of the control at $t=0$.
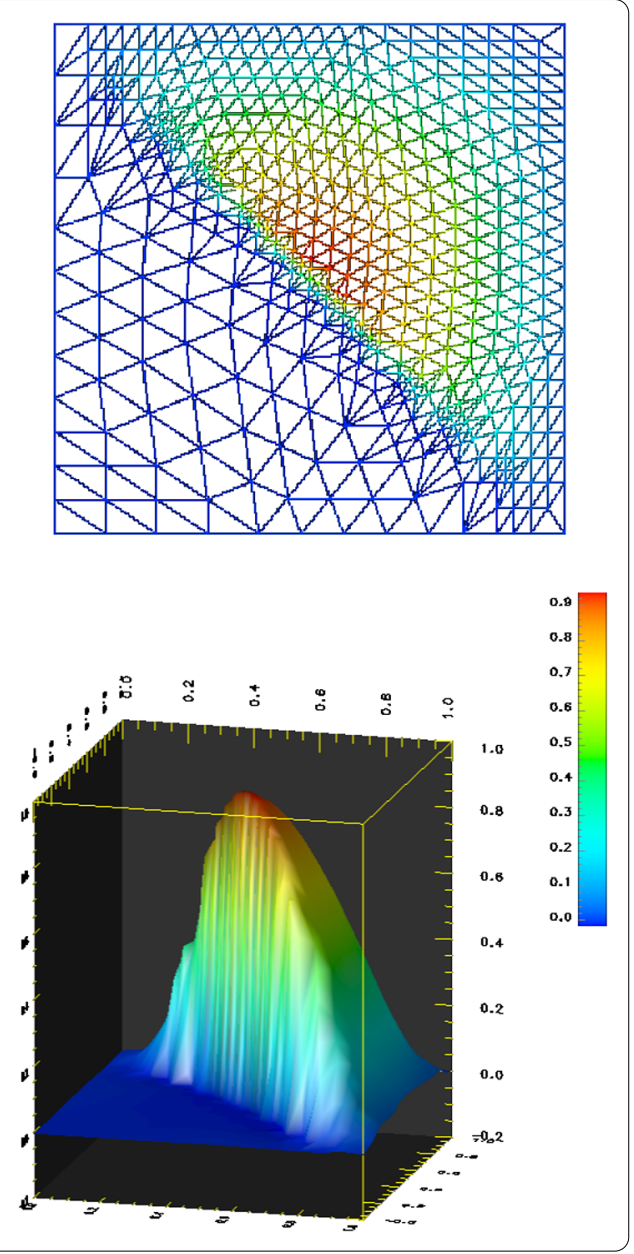

is $L^{2}\left(0, T ; H^{1}(\Omega)\right)$, and $L^{\infty}\left(L^{2}\right)$ is $L^{\infty}\left(0, T ; L^{2}(\Omega)\right), N$ is the number of nodes in the mesh, $S$ is the number of sides in the mesh, and $E$ is the number of elements in the mesh.

All data of the error estimates we obtain in the following tables are in norms of $L^{2}\left(0, T ; L^{2}(\Omega)\right), L^{2}\left(0, T ; H^{1}(\Omega)\right)$, and $L^{\infty}\left(0, T ; L^{2}(\Omega)\right)$. We note that, on the adaptive mesh, fewer nodes, sides, elements, and DOFs in the state variables are used. Since repeatedly solving the state and the costate equations is the main computational work in computing the control problem, we can save much computation work by using the adaptive mesh.

In Figures 2-4, we present the adaptive mesh for the control, state, and costate at different times. 
Figure 3 Adaptive mesh for the state and the approximation value of the state at $t=1$.
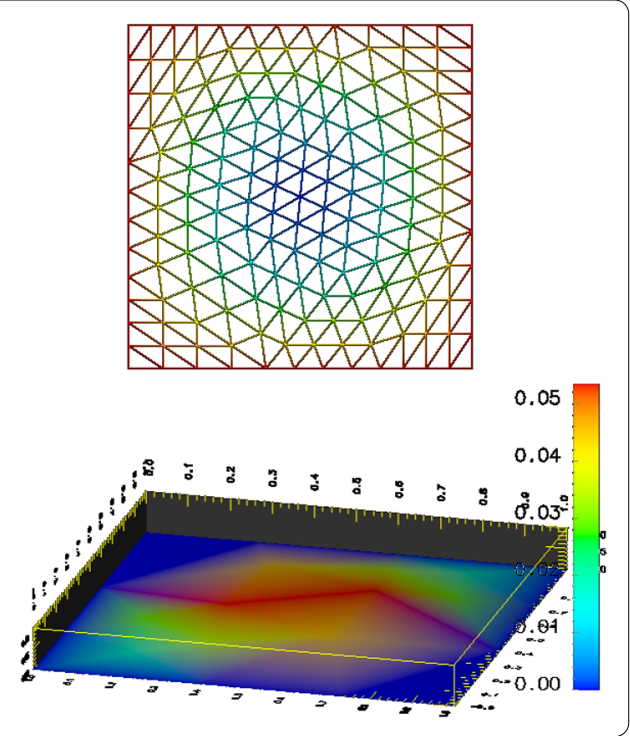

Figure 4 Adaptive mesh for the costate and the approximation value of the costate at $t=0$.
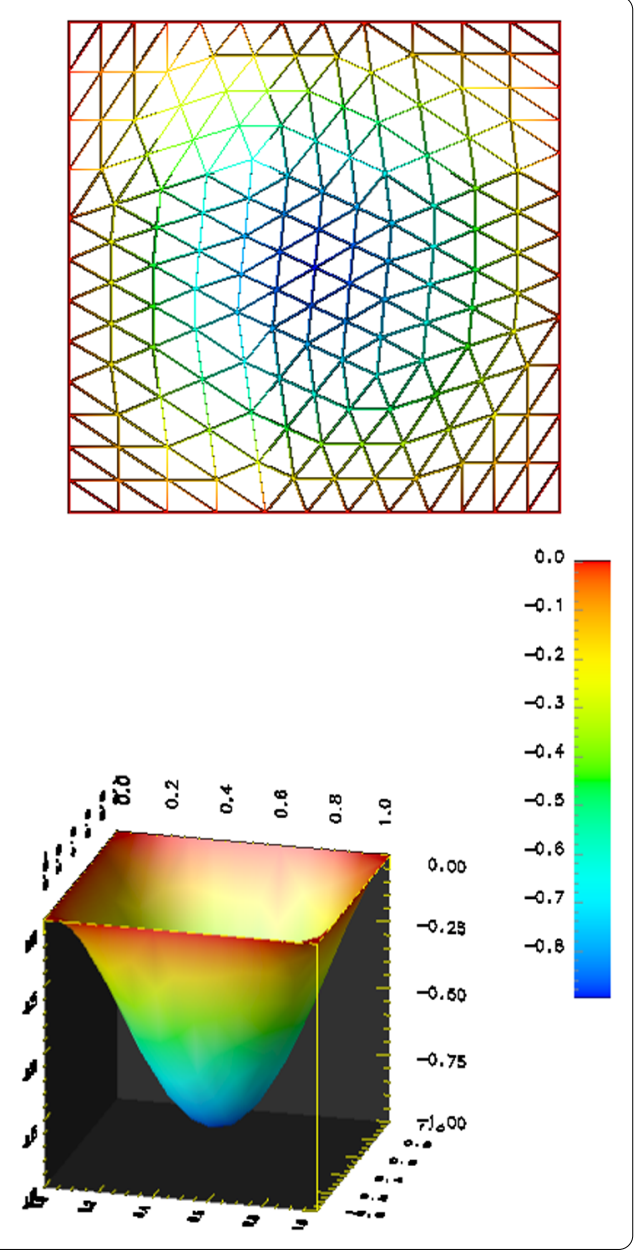


\subsection{Conclusions}

In this paper, a quadratic optimal control problem governed by a linear quasi-parabolic integro-differential equation and its adaptive finite element approximation are investigated for the first time. First, we present weak formulations for the control problem and optimal conditions and establish the full-discrete finite element approximation and the backward Euler scheme for the space and time approximation for optimal control problem. Then equivalent a posteriori error estimators with lower and upper bounds for both state and control approximation are derived. We use these indicators in our adaptive multimesh finite element schemes. Numerical experiments illustrate that the adaptive multimeshes can indeed significantly reduce computational work for this type of optimal control problems.

\section{Competing interests}

The author declares that they have no competing interests.

\section{Acknowledgements}

The author would like to express his thanks to the referees for their valuable comments and suggestions. This work is supported by National Natural Science Foundation of China (Grant 11501326).

Received: 5 January 2016 Accepted: 13 June 2016 Published online: 24 June 2016

\section{References}

1. Cui, S: Global solutions for a class of nonlinear integro-differential equations. Acta Math. Appl. Sin. 16(2), 191-200 (1993)

2. Cui, X: Sobolev-Volterra projection and numerical analysis of finite element methods for integro-differential equations. Acta Math. Appl. Sin. 24(3), 441-455 (2001)

3. Lions, JL: Optimal Control of Systems Governed by Partial Differential Equations. Springer, Berlin (1971)

4. Alt, W: On the approximation of infinite optimisation problems with an application to optimal control problems. Appl. Math. Optim. 12, 15-27 (1984)

5. Falk, FS: Approximation of a class of optimal control problems with order of convergence estimates. J. Math. Anal. Appl. 44, 28-47 (1973)

6. French, DA, King, JT: Approximation of an elliptic control problem by the finite element method. Numer. Funct. Anal. Optim. 12, 299-314 (1991)

7. Malanowski, K: Convergence of approximations vs. regularity of solutions for convex control constrained, optimal control systems. Appl. Math. Optim. 8(8), 69-95 (1982)

8. Neittaanmaki, P, Tiba, D: Optimal Control of Nonlinear Parabolic Systems: Theory, Algorithms and Applications. Dekker, New York (1994)

9. Pironneau, O: Optimal Shape Design for Elliptic Systems. Springer, Berlin (1984)

10. Tiba, D: Lectures on the Optimal Control of Elliptic Equations. University of Jyvaskyla Press, Jyvaskyla (1995)

11. Tiba, D, Tröltzsch, F: Error estimates for the discretization of state constrained convex control problems. Numer. Funct. Anal. Optim. 17, 1005-1028 (1996)

12. Hermann, B, Yan, NN: Finite element methods for optimal control problems governed by integral equations and integro-differential equations. Numer. Math. 101, 1-27 (2005)

13. Shen, W, Ge, L, Yang, D: Finite element methods for optimal control problems governed by linear quasi-parabolic integro-differential equations. Int. J. Numer. Anal. Model. 10(3), 536-550 (2013)

14. Liu, WB, Yan, NN: Adaptive Finite Elements Methods for Optimal Control Problem Governed by PDEs. Sciences Press, Beijing (2008)

15. Becker, R, Kapp, H, Rannacher, R: Adaptive finite element methods for optimal control of partial differential equations. SIAM J. Control Optim. 39, 113-132 (2000)

16. Becker, R, Rannacher, R: An optimal control approach to a posteriori error estimation. Acta Numer. 10, 1-102 (2001)

17. Li, R, Liu, WB, Ma, HP, Tang, T: Adaptive finite element approximation of elliptic optimal control. SIAM J. Control Optim. 41, 1321-1349 (2002)

18. Liu, WB, Yan, NN: A posteriori error analysis for convex distributed optimal control problems. Adv. Comput. Math. 15, 285-309 (2001)

19. Liu, WB, Tiba, D: Error estimates for the finite element approximation of a class of nonlinear optimal control problems. Numer. Funct. Anal. Optim. 22, 953-972 (2001)

20. Liu, WB, Yan, NN: A posteriori error estimates for optimal boundary control. SIAM J. Numer. Anal. 39, 73-99 (2001)

21. Shen, $W, G e, L$, Yang, D, Liu, W: A priori error estimates of finite element methods for linear parabolic integro-differential optimal control problems. Adv. Appl. Math. Mech. 6(5), 552-569 (2014)

22. Lions, JL, Magenes, E: Non Homogeneous Boundary Value Problems and Applications. Springer, Berlin (1972)

23. Shen, W, Ge, L, Yang, D, Liu, W: Sharp a posteriori error estimates for optimal control governed by parabolic integro-differential equations. J. Sci. Comput. 65(1), 1-33 (2015)

24. Scott, LR, Zhang, SY: Finite element interpolation of nonsmooth functions satisfying boundary conditions. Appl. Math. Optim. 54, 483-493 (1990)

25. Ciarlet, PG: The Finite Element Method for Elliptic Problems. North-Holland, Amsterdam (1978)

26. Verfurth, R: A Review of a Posteriori Error Estimation and Adaptive Mesh Refinement. Wiley/Teubner, London (1996)

27. Li, R: On multi-mesh h-adaptive algorithm. J. Sci. Comput. 24(3), 321-341 (2005) 